\title{
WestVirginiaUniversity
}

THE RESEARCH REPOSITORY @ WVU

Graduate Theses, Dissertations, and Problem Reports

2011

\section{Potential Energy Surface of Urea}

\author{
Xiaojian Mao \\ West Virginia University
}

Follow this and additional works at: https://researchrepository.wvu.edu/etd

\section{Recommended Citation}

Mao, Xiaojian, "Potential Energy Surface of Urea" (2011). Graduate Theses, Dissertations, and Problem Reports. 4750.

https://researchrepository.wvu.edu/etd/4750

This Dissertation is protected by copyright and/or related rights. It has been brought to you by the The Research Repository @ WVU with permission from the rights-holder(s). You are free to use this Dissertation in any way that is permitted by the copyright and related rights legislation that applies to your use. For other uses you must obtain permission from the rights-holder(s) directly, unless additional rights are indicated by a Creative Commons license in the record and/ or on the work itself. This Dissertation has been accepted for inclusion in WVU Graduate Theses, Dissertations, and Problem Reports collection by an authorized administrator of The Research Repository @ WVU.

For more information, please contact researchrepository@mail.wvu.edu. 


\title{
Potential Energy Surface of Urea
}

\author{
Xiaojian Mao \\ A dissertation submitted to the \\ Eberly College of Arts and Sciences \\ at West Virginia University \\ in partial fulfillment of the requirements \\ for the degree of \\ Doctor of Philosophy \\ in \\ Chemistry
}

Approved by

Charles Jaffé, Ph. D., Chair

Alfred H. Stiller, Ph. D.

Ken Showalter, Ph. D.

Terry Gullion, Ph. D.

John Penn, Ph. D.

C. Eugene Bennett Department of Chemistry

Morgantown, West Virginia

2011

Key word: urea; potential energy surface; ab initio 


\title{
Abstract \\ Potential Energy Surface of Urea
}

\author{
Xiaojian Mao
}

Using MP2 methods with aug-cc-pVDZ basis set, the inversion and rotation potential energy surface (PES) of urea molecule were plotted and fitted into polynomials. Important conformations on the PES were located and characterized. Their connecting paths were examined. Both inversion and rotation energy barriers were calculated. It was showed there could exist more than one internal rotation path for a floppy molecule. The topology of the PES were characterized using graphical description and symmetry analysis. 
To my wife, Jing Zhu,

and my parents,

whose love is always with me 


\section{Acknowledgements}

I would like to express my sincere appreciation and great gratitude to my advisor Dr. Charles Jaffé. This work would not have been possible without his unfaltering support and encouragement. His inspiring encouragement, his guidance on this research, and his patience are the most important factors which help me through the graduate study. He is not only the advisor, but also a good friend. His help is not limited in chemistry and is extended to my daily life in Morgantown.

Appreciation is also due to my committee members, Dr. Alfred H. Stiller, Dr. Ken Showalter, Dr. Terry Gullion, and Dr. John Penn for their precious time, guidance, suggestions, help and encouragement.

I would also like to thank many people for their friendship and assistance in the chemistry department during these years while it is hard to name every individual.

My very special appreciation goes to my wife, Jing Zhu, and my parents. Their love are the key to support me to overcome the obstacles during all these years.

Finally, many thanks to the Department of Chemistry at West Virginia University for providing me uninterrupted financial support. 


\section{Contents}

List of Figures

List of Tables

1 Introduction 1

1.1 Urea as a protein denaturing agent $\ldots \ldots \ldots \ldots$. . . . . . . .

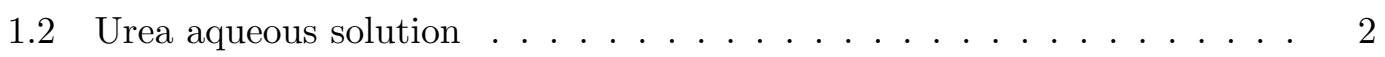

1.3 Structure of urea molecules $\ldots \ldots \ldots \ldots$

1.4 Goal of this research . . . . . . . . . . . . 5

2 Method and Model

2.1 Coupling of Inversion and Rotation . . . . . . . . . . . . . . 7

2.2 Inversion and Rotation Variables . . . . . . . . . . . . . . . . 9

2.3 Plotting PES . . . . . . . . . . . . . . . . . 11

2.4 Computational Details . . . . . . . . . . . . . . . . . 11

2.4 .1 GAMESS ........................ 11

2.4 .2 Basis Set . . . . . . . . . . . . . 12

2.4.3 Aug-cc-pVDZ Basis Set . . . . . . . . . . . . . 12

2.4.4 Calculation Level . . . . . . . . . . . . . . . . . . . 14

3 Two-dimensional Inversion Potential Energy Surface 
3.1 Inversion PES . . . . . . . . . . . . . . . 15

3.1 .1 Important conformations . . . . . . . . . . . . . . . 15

3.1.2 Hybridization of nitrogen atoms . . . . . . . . . 17

3.1 .3 Inversion Barrier . . . . . . . . . . . . . . . . 20

3.1 .4 Population Map . . . . . . . . . . . . . . . . 20 20

3.1 .5 Dipole Moment . . . . . . . . . . . . . . . . . . 21

3.2 Fitting inversion $\mathrm{PES} \ldots \ldots \ldots \ldots \ldots$

3.3 Normal Mode Analysis . . . . . . . . . . . . . . . . . . . . . . . 26

3.3.1 Degrees of Freedom . . . . . . . . . . . . . . 26

3.3 .2 Kinetic Energy Terms . . . . . . . . . . . . . . . 26

3.3 .3 Potential Energy Terms . . . . . . . . . . . . . . 28

3.3.4 Normal mode coordinates . . . . . . . . . . . . . . 30

3.3.5 Rank-2 Saddle ... . . . . . . . . . . . . . . 31

3.4 Two-dimensional Schrödinger Equation . . . . . . . . . . . . 33

4 Four-dimensional Rotation Potential Energy Surface 39

4.1 Internal rotation $\mathrm{PES} \ldots \ldots \ldots \ldots$

4.2 Important conformations . . . . . . . . . . . . . . . . . . . 39

4.3 Dipole moments . . . . . . . . . . . . . . . 42

4.4 Internal rotation barrier $\ldots \ldots \ldots \ldots \ldots \ldots$

4.4 .1 Early results . . . . . . . . . . . . . . 43

4.4 .2 Internal rotation barrier . . . . . . . . . . . . 43

4.4 .3 Conjugation . . . . . . . . . . . . . . . . . 44

4.4.4 Rotation barrier of floppy molecules . . . . . . . . . 44

4.5 Fitting rotation PES . . . . . . . . . . . . . . . 45

5 Symmetry Analysis 46

5.1 Complete Permutation-inversion Group . . . . . . . . . . . . . 46 
5.2 Subsurfaces of PES . . . . . . . . . . . . . . . 46

5.3 Longuet-Higgins Group . . . . . . . . . . . . . . . . . . 47

5.4 Longuet-Higgins Group of Urea . . . . . . . . . . . . . . . . . 48

5.5 Tunneling . . . . . . . . . . . . . . . . . . . . . 49

5.6 Electric Dipole Selection Rules . . . . . . . . . . . . . . . . . . 53

5.7 Symmetry of inversion PES . . . . . . . . . . . . 5 54

5.8 Symmetry of rotation PES . . . . . . . . . . . . . . 5 54

5.9 Graphical Description . . . . . . . . . . . . . . . . 55

5.10 Graphical Description of Urea PES . . . . . . . . . . . . 555

6 Conclusion

\begin{tabular}{ll} 
Appendices & 60 \\
\hline
\end{tabular}

Appendix A Potential energy of urea fitting around conformation D 61

Appendix B Coefficients of fitted rotation PES of urea 63

References 


\section{List of Figures}

1.1 Structure of urea in solid state, which shows an extensive hydrogen bond-

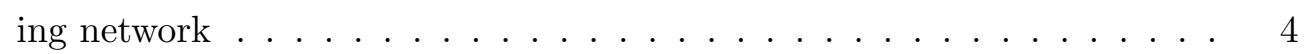

1.2 Structure of urea in gas state: solid NH bonds come out of the page and the $\mathrm{NH}$ dashed bonds go into the page . . . . . . . . . . 5

2.1 Atom numbering for urea molecule . . . . . . . . . . . . 8

3.1 Inversional potential energy surface of urea molecule . . . . . . . . . 16

$3.2 \quad$ A - global minimum, $\mathrm{C}_{2} \ldots \ldots \ldots \ldots$

3.3 B - local minimum $\mathrm{C}_{s} \ldots \ldots \ldots \ldots \ldots$

$3.4 \mathrm{C}-\operatorname{rank}-2$ saddle, $\mathrm{C}_{2 v} \ldots \ldots \ldots \ldots$

3.5 D - rank-1 saddle, $\mathrm{C}_{1} \ldots \ldots \ldots$. . . . . . . . . . . . . . .

3.6 Population map . . . . . . . . . . . . . . . . . 22

3.7 Mesomeric structures of urea molecule . . . . . . . . . . . . . . 23

3.8 Ground state wave function of urea molecule from inversion potential . . 35

3.9 Contour map of ground state wave function of urea molecule from inversion potential $\ldots \ldots \ldots \ldots \ldots \ldots \ldots \ldots$

3.10 First excited state wave function of urea molecule from inversion potential 36

3.11 Contour map of first excited state wave function of urea molecule from inversion potential .................... . . . . . . . . . 
3.12 Second excited state wave function of urea molecule from inversion potential ........................ 37

3.13 Contour map of second excited state wave function of urea molecule from inversion potential $\ldots \ldots \ldots \ldots \ldots \ldots \ldots \ldots$

3.14 Third excited state wave function of urea molecule from inversion potential 38

3.15 Contour map of third excited state wave function of urea molecule from inversion potential $\ldots \ldots \ldots \ldots \ldots \ldots \ldots$

4.1 Rotational potential energy surface of urea molecule . . . . . . . . 40

4.2 E-rank-2 saddle $\mathrm{C}_{2 v} \ldots \ldots \ldots \ldots$

$4.3 \mathrm{~F}-$ rank-2 saddle, $\mathrm{C}_{s} \ldots \ldots \ldots$

$4.4 \mathrm{G}-$ rank-2 saddle, $\mathrm{C}_{2 v} \ldots \ldots \ldots \ldots \ldots$

$4.5 \mathrm{H}-\operatorname{rank}-1$ saddle, $\mathrm{C}_{s} \ldots \ldots \ldots \ldots$

4.6 I - rank-2 saddle $\mathrm{C}_{s} \ldots \ldots \ldots \ldots$

5.1 Eight energetically equivalent global energy minimum conformations of urea molecule which are accessible to each others. . . . . . . . . . . 47

5.2 Atom numbering for urea molecule's permutation-inversion operation . . 48

5.3 Elements of urea's permutation group: cont. . . . . . . . . . . 50

5.4 Elements of urea's permutation group: contd. . . . . . . . . . . . 51

5.5 Graphic description of connections on PES of urea molecule . . . . . . 56

5.6 Inversion paths on PES of urea molecule . . . . . . . . . . . 57

5.7 One-side rotation paths on PES of urea molecule . . . . . . . . 57

5.8 Two-side rotation paths on PES of urea molecule $\ldots \ldots \ldots$ 


\section{List of Tables}

2.1 Urea geometry optimization using different basis sets . . . . . . . . 13

3.1 Important conformations in inversion PES of urea molecule . . . . . . 18

3.2 Out-of plane angles of different urea molecule conformations in its inver-

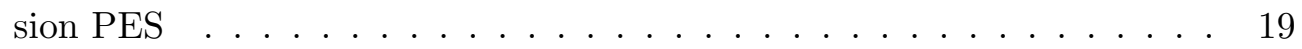

3.3 Dipole moment of urea molecules . . . . . . . . . . . . . . 2 21

3.4 Coefficients of fitted inversion PES of urea . . . . . . . . 25

3.5 Conventional internal coordinates of urea molecule . . . . . . . . 26 26

3.6 Energy levels of urea molecule's inversion potential . . . . . . . . . . 33

4.1 Out-of plane angle of different urea molecule conformations in its rota-

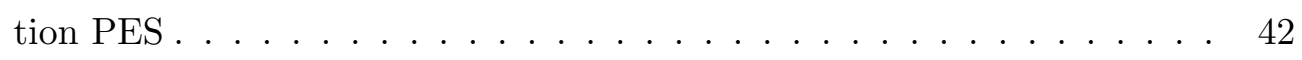

4.2 Dipole moments of important conformations in rotation PES of urea molecule . . . . . . . . . . . . . . . . 42

4.3 Important conformations in rotation PES of urea molecule . . . . . . . 43

4.4 CN bond lengths of important conformations in rotation PES of urea molecule . . . . . . . . . . . . . . . . 44

5.1 Character Table of Longuet-Higgins Group of Urea While Inversion and

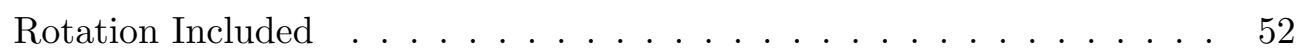

5.2 Reducible representation of symmetric and antisymmetric levels . . . . 53 
5.3 Character Table of Longuet-Higgins Group of Urea While Only Inversion Included . . . . . . . . . . . . . . . . . 54

5.4 Character Table of Longuet-Higgins Group of Urea While Only Rotation Included . . . . . . . . . . . . . . . . 55

5.5 Number of important conformations in PES of urea molecule . . . . . 56

A.1 Coefficients of fitted PES of urea around conformation D . . . . . 62 62

B.1 Coefficients of fitted rotation PES of urea . . . . . . . . . . . 64 


\section{Chapter 1}

\section{Introduction}

Urea was the first organic compound prepared outside of a living organism in history. It was synthesized from ammonium sulfate and lead cyanate by Wöhler in 1828 [1]. This is marked as the demise of the vital force theory and the beginning of modern organic chemistry. Since then it has been the subject of interest in almost every branch of fundamental and applied chemistry research field.

\subsection{Urea as a protein denaturing agent}

Although urea is structurally simple and has been investigated for such a long time, many of its properties are still not understood and draw a great amout of interest in many fields of chemistry. Among them, its well-known but as yet unexplained denaturation effects [2,3] in a variety of peptide and protein like structures have triggered a great deal of research [4-11] in recent years. By adding to and removing from urea solution, proteins can switch their conformations between native structures and denatured random coil states.

The manner in which urea affects protein is still unclear. Some believe it is related to the hydrogen bonding ability of urea to the peptide backbone [5, 6, 8, 11], others 
believe it is related to the water structure change due to urea molecules $4,7,12$. In former case, urea molecules will break the original hydrogen bondings of protein like structures, and new hydrogen bondings will form between urea molecules and protein molecules. In later case, urea will change the water "icelike" structure, therefore trigger the change of activity of peptide groups.

Although it is still unclear which hypothesis is correct, urea molecule's hydrogen bonding ability and interaction with water molecules play very important roles in its denaturation ability.

\subsection{Urea aqueous solution}

Urea aqueous solution has been an interesting topic for decades due to the curious manner in which urea molecule affects aqueous solution. "Urea is considered a solute class of its own, because of the possibly unique characteristics of its interactions with water." 13

The role of urea in water structure is still an open question. Raman spectral method [14] and ultrasonics study [15 have showed evidence of urea molecules as a water structure breaker. Different models were proposed to interpreted the experimental data $4,16,17]$.

On the other hand, heat capacity and NMR data 12,18 suggested urea molecules neither promote nor destruct the water structure. Infrared spectra method [6, 10], neutron diffraction data [9] and molecular dynamics calculations [19] also support the idea that urea molecules could fit into the water structure without any considerable distortion. Urea molecule, while dissolving in water, might provide a similar range of geometries and keep a similar number of hydrogen bondings (relative to the water molecules it replaces in water structure), therefore, water's structure is kept unchanged.

In order to fit into water structure, urea molecule has to have "correct" geometry 
of structure. All these studies of how urea molecule affects water structure lead to another important question: what is the geometry structure of urea molecule in aqueous solution?

\subsection{Structure of urea molecules}

Because of urea molecule's simplicity of structure, it is one of the first organic compounds to be determined by X-ray crystallographic method [20 22], and later confirmed and supplemented by neutron diffraction measurements 2326 . The structure of urea molecule in solid state is well-known today as a planar structure (Fig. 1.1). Its eight atoms, including atoms from the two amide groups it contains, are in a same plane. The planar structure is due to an extensive hydrogen bonding network existing in crystal structure of urea: each urea molecule is hydrogen bonded to its six neighbors.

It was only in nineties that quantum mechanic calculations $27-29]$ showed urea molecule in gas state should be nonplanar. Instead of all eight atoms lie in a same plane, the two amino groups actually adopt a pyramidal structure (Fig. 1.2). The microwave spectrum 30 and matrix isolation study data 31] were re-examed to support the calculations. And this nonplanar structure was confirmed later by microwave spectra data 32 and infrared spectra data 33 .

A lot of effort has been put into the study of geometry of urea molecules in aqueous solution 17, 18, 34, 37. Although the structure of urea molecule in solid and gas state are generally accepted today, the geometry of urea molecules in aqueous solution is still an open question [36]. The answer to this question is crucial to determine its action on proteins through its influence on water structure. 


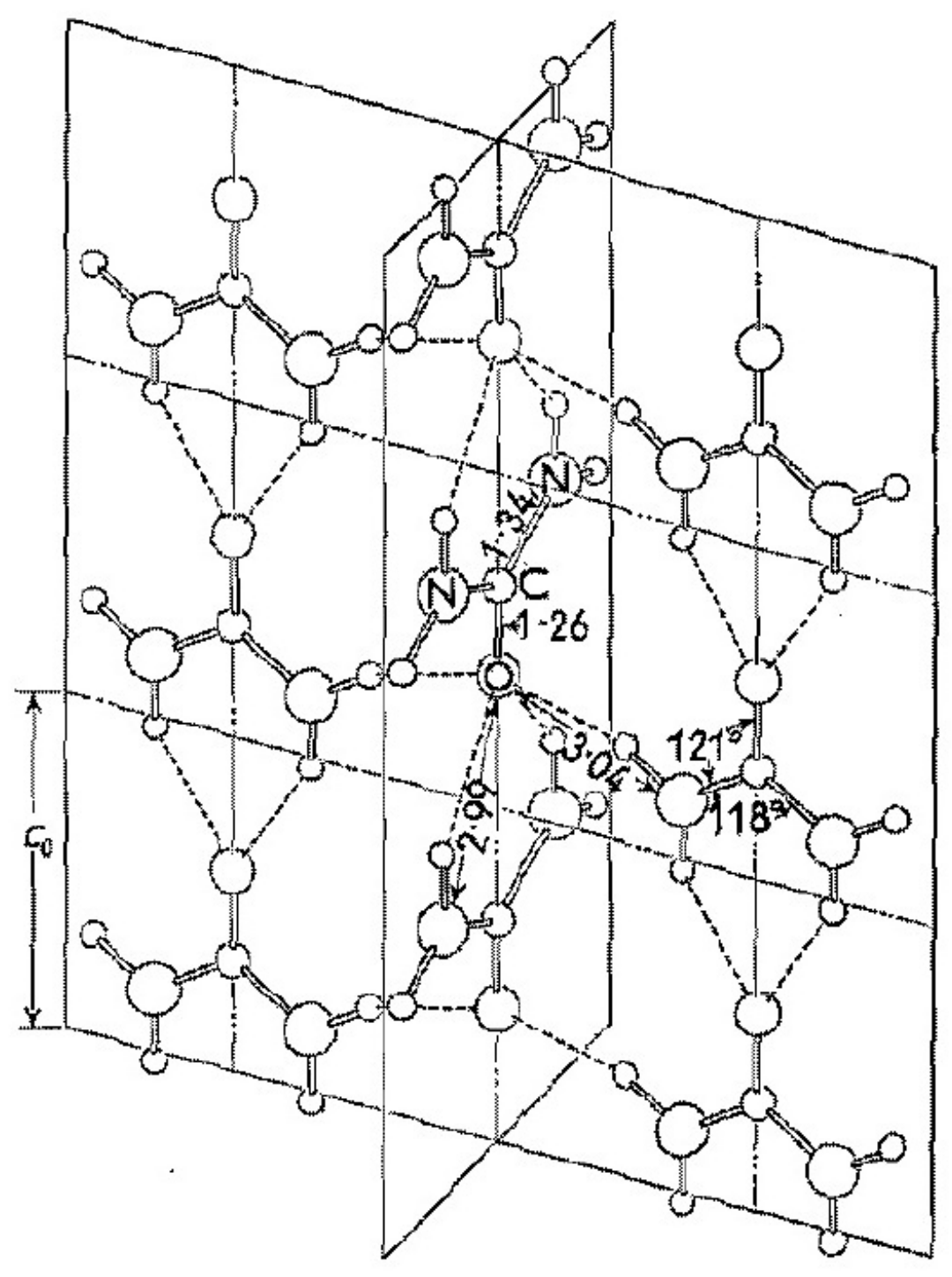

Figure 1.1: Structure of urea in solid state, which shows an extensive hydrogen bonding network - Figure adapted from figure 1, Vaughan, P. and Donohue, J., Acta Crystallographica, 1957, 5, 530-535. 


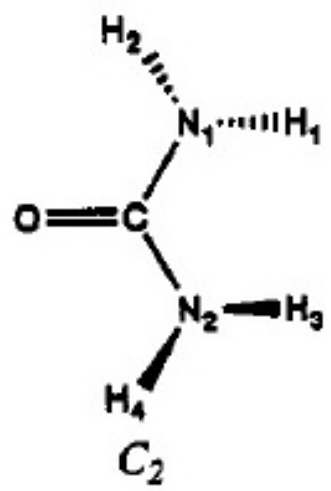

Figure 1.2: Structure of urea in gas state: solid NH bonds come out of the page and the NH dashed bonds go into the page - Figure adapted from figure 1, Dixon, D. A. \& Matsuzawa, N., Journal of Physical Chemistry, 1994, 98, 3967-3977.

\subsection{Goal of this research}

From above discussion it is clear that two properties of urea molecule are essential to understand its protein denaturation effect: its hydrogen bonding ability and molecular geometry structure. Actually, its hydrogen bonding ability is also closely related to its geometry structure. Its planar structure in solid state is due to the existence of extensive hydrogen bonding network, and quantum mechanics calculations did show that urea molecules, while forming dimer through hydrogen bonding, tend to be more planar than in monomers $37-39$.

The structure of urea in an environment, such as water or a protein surface, is a very fundamental question and has not been definitely resolved. Knowledge of the shapes and energetics of urea molecules is essential for understanding this question.

It is amazing that a small geometry change in molecular structure could have large influences on molecular properties. To understand the effects better, it is of importance to investigate the flexibility of urea molecule and its topology of potential energy surface (PES). Most energy minima and saddles have been reported in several studies 28,29 , 32, 38, 40, 41. However, in these studies only single points in the PES have been 
investigated. The incentive for this study was to help understanding urea molecule's property by investigating the whole topology of urea molecule's PES. 


\section{Chapter 2}

\section{Method and Model}

\subsection{Coupling of Inversion and Rotation}

Urea molecule is considered as a typical floppy molecule due to its ability to switch between planar and non-planar structure. A floppy molecule is a molecule which possesses more than one equilibrium configuration and the energy barrier between these configurations are low enough for it to switch between them. There are mainly two types of motions which could occur within a floppy molecule: internal inversion and rotation, and they both exist within urea molecules.

Both experimental [32 and theoretical [27] results have shown that urea molecule in gas state is a non-planar structure. The hybridisation of the two nitrogen atoms is $s p^{3}$ rather than $s p^{2}$ in non-planar structures. The two amino groups are pyramidal and these trigonal pyramid geometry could undergo nitrogen inversion to turn the hydrogen atoms in and out (Fig. 2.1). This is the internal inversion motion of urea molecules.

Quantum mechanics calculations also showed that the rotational barrier around CN bonds are low (around $11 \mathrm{kcal} / \mathrm{mol}$ ) [40 42] enough for urea molecules to make the internal rotation feasible.

The two nitrogen inversions and two $\mathrm{CN}$ bond rotations together make urea a 
complicated floppy molecule, although it is structurely simple. These two inversions and two rotations are coupled together. Due to this coupling, a problem which has never been noticed before arises when using the traditional way to calculate and plot rotational barrier of urea.

In a traditional way, to calculate the relaxed $\mathrm{C}_{1}-\mathrm{N}_{3}$ rotational barrier of urea (Fig. 2.1), one will fix the dihedral angle $\mathrm{O}_{2} \mathrm{C}_{1} \mathrm{~N}_{3} \mathrm{H}_{6}$ and leave all other degrees of freedom relaxed to calculate the energy. By changing the fixed value of the dihedral angle $\mathrm{O}_{2} \mathrm{C}_{1} \mathrm{~N}_{3} \mathrm{H}_{6}$ from 0 to 360 , a rotational energy barrier can be plotted.

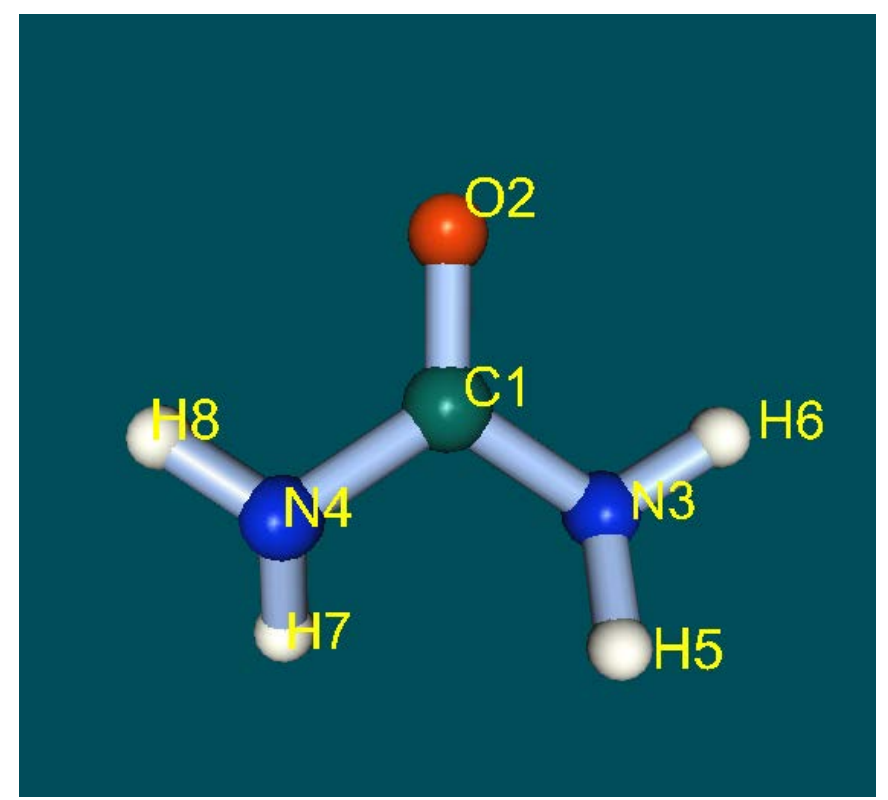

Figure 2.1: Atom numbering for urea molecule - Plotted using Facio software

However, this traditional way to plot rotational barrier does not work for urea due to the existing coupling between rotation and inversion within urea molecule.

First, since the coordinate of the other hydrogen atom in the same amino group $\left(\mathrm{H}_{5}\right)$ is not fixed, this atom still can undergo inversion motion to make urea molecule reaches different energy minimum configurations. Inversion is interfering rotation during calculation. This problem is arising from using conventional internal coordinates (dihedral angle $\mathrm{O}_{2} \mathrm{C}_{1} \mathrm{~N}_{3} \mathrm{H}_{6}$ ) since the dihedral angle is actually a variable involving 
in both rotation and inversion motions. In order to avoid this interfering, instead of using conventional internal coordinates, new coordinates have to be designed to plot the rotational barrier.

Second, there is also interfering from the rotation and inversion motions of the other amino group $\left(-\mathrm{N}_{4} \mathrm{H}_{7} \mathrm{H}_{8}\right)$. Since this group is left relaxed during calculations, it can undergo inversion motion to reach different energy mimimum configurations. Depending on different configuration it chooses, different rotation path will arise. The traditional rotational barrier calculation will generate a multivalued plotting. This multivalue problem is arising from the coupling between motions of two amino groups. Any one-dimensional rotation barrier plotting of urea molecule can not avoid it. The rotation paths of urea molecule has to be presented at least in a two-dimensional plotting.

\subsection{Inversion and Rotation Variables}

For urea molecule (Fig. 2.1), the four heavy atoms (carbon, oxygen, and two nitrogen atoms) stay in the same plane for most of time, and it is the positions of four hydrogen atoms make the big difference of the molecular geometry. Actually, experimental data shows that the skeleton vibrations of urea molecule are not coupled with the amino groups deformation vibration [43]. Therefore, it is reasonable to only consider the positions of hydrogen atoms while investigating the flexibility of urea molecules.

In conventional internal coordinate system, the positions of these four hydrogen atoms are represented by four dihedral angles (Fig. 2.1):

$$
\begin{aligned}
\tau_{5} & =\text { dihedral angle } \mathrm{O}_{2} \mathrm{C}_{1} \mathrm{~N}_{3} \mathrm{H}_{5} \\
\tau_{6} & =\text { dihedral angle } \mathrm{O}_{2} \mathrm{C}_{1} \mathrm{~N}_{3} \mathrm{H}_{6} \\
\tau_{7} & =\text { dihedral angle } \mathrm{O}_{2} \mathrm{C}_{1} \mathrm{~N}_{3} \mathrm{H}_{7}
\end{aligned}
$$




$$
\tau_{8}=\text { dihedral angle } \mathrm{O}_{2} \mathrm{C}_{1} \mathrm{~N}_{3} \mathrm{H}_{8}
$$

A dihedral angle is the angle between two planes. Dihedral angle $\mathrm{O}_{2} \mathrm{C}_{1} \mathrm{~N}_{3} \mathrm{H}_{5}$ is defined as the angle between two planes which are defined by $\mathrm{O}_{2}, \mathrm{C}_{1}, \mathrm{~N}_{3}$ atoms and $\mathrm{C}_{1}, \mathrm{~N}_{3}, \mathrm{H}_{5}$ atoms respectively.

However, just as discussed in section 2.1, each of these dihedral angles actually involves in both rotation and inversion motions. In order to seperate the coupling between rotation and inversion, a set of new coordinates is used:

$$
\begin{aligned}
\theta_{1} & =\frac{\tau_{5}+\tau_{6}}{2} \\
\theta_{2} & =\frac{\tau_{7}+\tau_{8}}{2} \\
\tau_{1} & =\tau_{5}-\tau_{6} \\
\tau_{2} & =\tau_{7}-\tau_{8}
\end{aligned}
$$

Rotation coordinates $\theta_{1}$ and $\theta_{2}$ represent the rotation motions of two amino groups. When the amino groups rotate around CN bonds, these two variables will change from 0 to 360 .

Inversion coordinates $\tau_{1}$ and $\tau_{2}$ are the differences between two dihedral angles in a same amino group. When the amino groups undergo inversion motions, switching between trigonal pyramid structures, the variables $\tau_{1}$ and $\tau_{2}$ will change their values around 180. These variables represent how much the geometries of amino groups are out-of-plane near the planar structures, and can be considered as inversion coordinates.

With these new inversion and rotation coordinates, the inversion and rotation potential energy surface (PES) of urea can be calculated and plotted. 


\section{$2.3 \quad$ Plotting PES}

To plot inversion potential energy surface of urea, urea molecule's energy were calculated (computational details descripted in chapter 2.4) with two inversion variables $\tau_{1}$ and $\tau_{2}$ changing from 100 to 260. At each point, the values of $\tau_{1}$ and $\tau_{2}$ were fixed, but all other 16 degrees of freedom of urea molecule were left relaxed. Urea molecule's geometry was then optimized and energy was calculated. Each energy generated a point in the PES, and a contour plot was done to represent the inversion PES of urea.

To plot rotation potential energy surface of urea, urea molecule's energy were calculated with two rotation variables $\theta_{1}$ and $\theta_{2}$ changing from 0 to 360 . At each point, the values of $\theta_{1}$ and $\theta_{2}$ were fixed, but all other 16 degrees of freedom of urea molecule were left relaxed. Urea molecule's geometry was then optimized and energy was calculated. Each energy generated a point in the PES, and a contour plot was done to represent the rotation PES of urea.

\subsection{Computational Details}

All calculations were carried out using the GAMESS (The General Atomic and Molecular Electronic Structure System) program [44] with the correlation-consistent aug-ccpVDZ basis set [45, 46] at second-order Möller-Plesset perturbation theory (MP2) 47] level.

\subsubsection{GAMESS}

GAMESS (The General Atomic and Molecular Electronic Structure System) is a general ab initio quantum chemistry package [44], maintained by the members of the Gordon research group at Iowa State University.

GAMESS can perform all kinds of general computational chemistry calculations, including Hartree-Fock, density functional theory (DFT), and Multi-configurational 
self-consistent field (MCSCF). Correlation corrections after these SCF calculations can be estimated by configuration interaction (CI), second order Mïler-Plesset perturbation theory, and coupled cluster theory. Solvent effect can also be considered using quantum mechanics/molecular mechanics through discrete effective fragment potentials or continuum models.

\subsubsection{Basis Set}

Choosing basis set is crucial for urea structure optimization calculations. In history, before nineties, urea structure was calculated as a planar structure due to using small basis sets. The topology of PES of urea could change a lot if using inappropriate basis set. The planar structure, which should be a rank-2 saddle, could be calculated as an energy minimum using inappropriate basis set!

To find out the appropriate basis set to use, different basis sets were used to optimize the structure of urea molecule and compared with experimental data (Table 2.1).

It can be seen from the data that both polarization functions and diffuse functions are important to be included in basis set when running calculations for urea molecule.

Polarization functions are higher angular momentum functions and they generally help to describe important charge polarization effects. Diffuse functions are functions with small exponents and they are important whenever loosely bound electrons are present. For urea molecule, the two lone pair electrons from nitrogen atoms is conjugated to $\pi$ orbital of CO bonding. Apparently, polarization effect is important within urea molecule, and an appropriate basis set should include both polarization functions and diffuse functions.

\subsubsection{Aug-cc-pVDZ Basis Set}

A set of the most widely used basis sets are correlation-consistent basis sets developed by Dunning and coworkers. They are designed to converge systematically to the 
ลิ

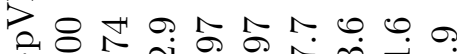

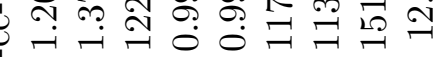

峞

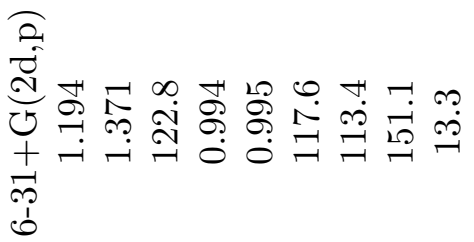

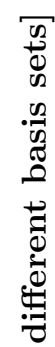

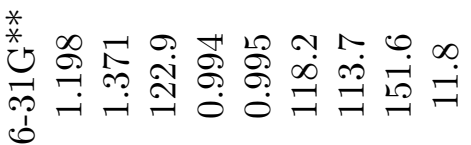

* 大

品

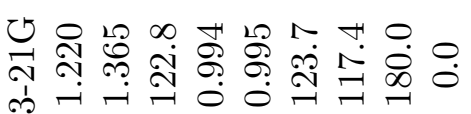

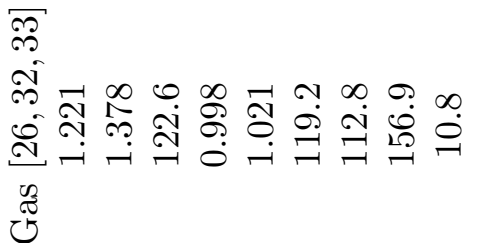

$4<<<\frac{0}{\pi}$

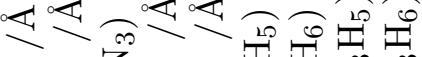

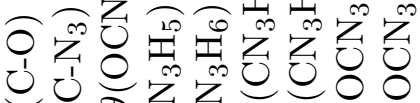

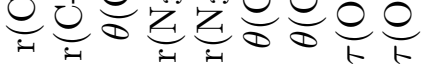


complete-basis-set limit. They include successively larger shells of polarization functions, and have become the current state of the art for post-Hartree-Fock calculations. Aug-cc-pVDZ basis set is the augmented version (with added diffuse functions) of the correlation-consistent polarized valence-only double-zeta basis set.

\subsubsection{Calculation Level}

Electron correlations are neglected in Hartree-Fock calculations. In HF treatment, electron-electron repulsion is handled by having each electron move in a smeared-out, average electrostatic field due to all the other electrons. Therefore, in HF treatment the probability that an electron will have a particular spatial coordinate at some moment is independent of the coordinates of the other electrons at that moment. This is not true in reality of course. A post-HF calculation should always be considered to correct the electron correlation when possible.

The Möller-Plesset (MP) treatment [47] of electron correlation is based on perturbation theory. Second order MP method (MP2) is a standard level used today in calculating small systems. 


\section{Chapter 3}

\section{Two-dimensional Inversion Potential Energy Surface}

\subsection{Inversion PES}

Urea molecule's energy were calculated (computational details described in section 2.4) with two inversion variables $\tau_{1}$ and $\tau_{2}$ (described in section 2.3 ) changing from $100^{\circ}$ to $260^{\circ}$. At each point, the values of $\tau_{1}$ and $\tau_{2}$ were fixed, but all other 16 degrees of freedom of urea molecule were left relaxed. Urea molecule's structure was then optimized and energy was calculated. Each energy generated a point in the PES. Totally 1800 points were calculated and a contour plot was done to represent the inversion PES of urea (Fig. 3.1).

\subsubsection{Important conformations}

Four most important conformations are labelled in Fig. 3.1 and listed in Fig. 3.2 - Fig. 3.5 .

conformation A is the global energy minimum. Agreed with previous work 27] it is a nonplanar structure with the $\mathrm{C}_{2}$ symmetry. Four heavy atoms lie in a same plane, 


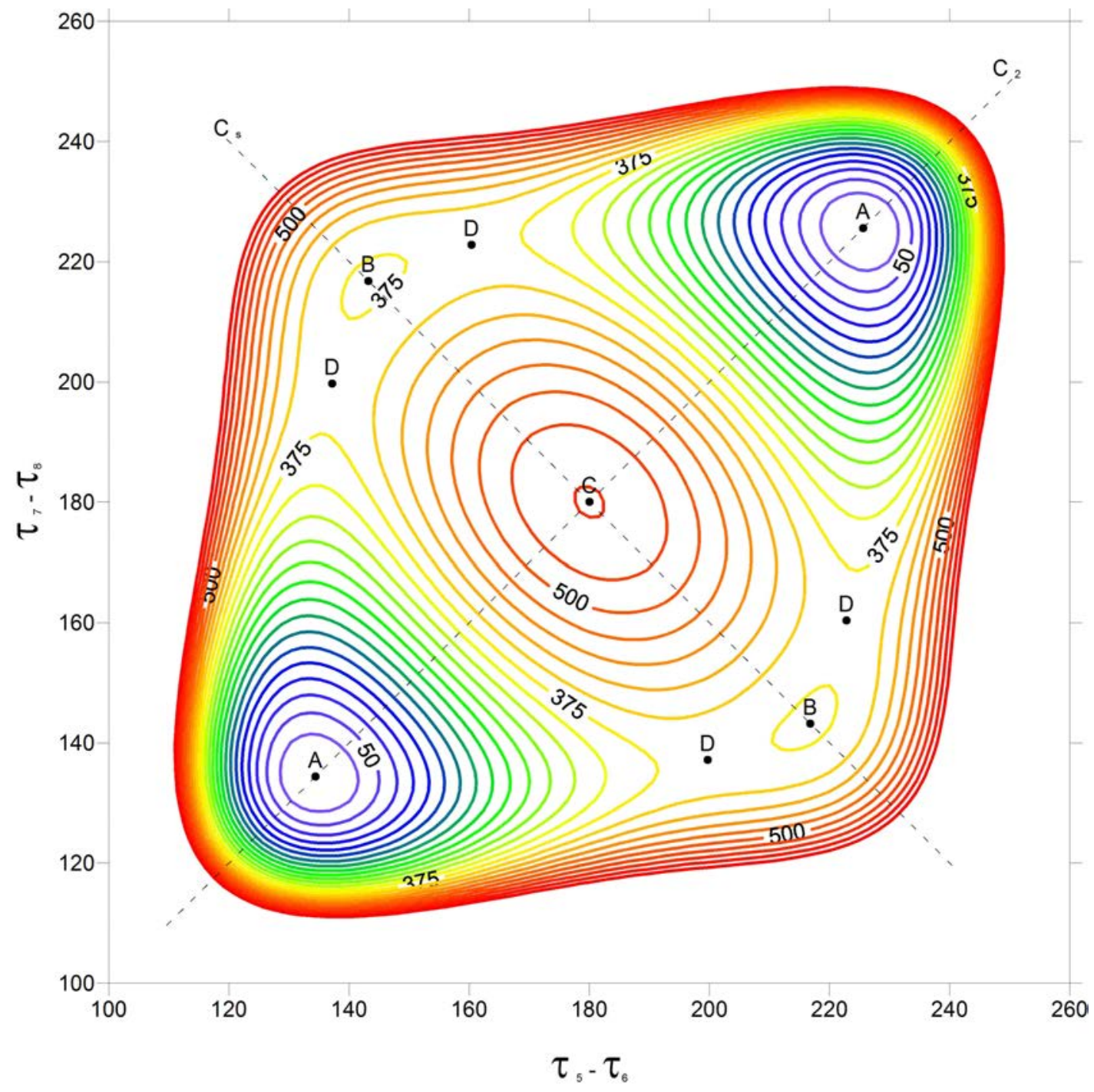

Figure 3.1: Inversional potential energy surface of urea molecule - unit in $\mathrm{cm}^{-1}$. 
two $-\mathrm{NH}_{2}$ group points to opposite direction and in two sides of that plane respectively. This is the conformation urea molecules adopt in gas state.

conformation B is the local energy minimum, with energy $1.06 \mathrm{kcal} / \mathrm{mol}$ higher than global energy minimum and with $\mathrm{C}_{s}$ symmetry. The two $-\mathrm{NH}_{2}$ group points to same direction and in the same side of four heavy atoms. The local energy minimum conformation connects with global energy minimum by inverting only one side of nitrogen trigonal pyramid structure.

conformation $\mathrm{C}$ is a rank-2 saddle. One of its decreasing normal mode coordinates leads to two global minima and another leads to two local minima. All eight atoms are lying in a same plane in this conformation. The symmetry is $\mathrm{C}_{2 v}$ and its energy is 1.58 $\mathrm{kcal} / \mathrm{mol}$ higher than global energy minimum. This is the conformation urea molecules adopt in solid state.

conformation D is a rank-1 saddle which connects global and local energy minimum. Its existence is in accord with Murrell-Laidler theorem [48], which predicts that there must exist a rank-1 saddle if a rank-2 saddle (conformation $\mathrm{C}$ in this case) is found to connect two minima (conformation A and B in this case). The only decreasing normal mode coordinate of this conformation leads to global minimum conformation and local minimum conformation respectively at its two ends. Its energy is $1.09 \mathrm{kcal} / \mathrm{mol}$ higher than global energy minimum.

\subsubsection{Hybridization of nitrogen atoms}

The geometry change of urea molecule's structure is closely related to hybridization of nitrogen atoms. When both nitrogen atoms take $s p^{2}$ hybridization, the urea molecule is in planar structure. With more $s p^{3}$ hybridization, the structure will be further from a planar geometry.

While in $s p^{3}$ hybridization, $-\mathrm{NH}_{2}$ group forms a trigonal pyramid and the sum of three angles is not equal to 360 . This difference between 360 and the sum is called 


\begin{tabular}{|c|c|c|c|c|}
\hline & Conformation A & Conformation B & Conformation C & Conformation D \\
\hline Symmetry & $\mathrm{C}_{2}$ & $\mathrm{C}_{s}$ & $\mathrm{C}_{2 v}$ & $\mathrm{C}_{1}$ \\
\hline Saddle Index & global minimum & local minimum & rank-2 saddle & rank-1 saddle \\
\hline Energy $/ \mathrm{kcal} / \mathrm{mol}$ & 0 & 1.06 & 1.58 & 1.09 \\
\hline $\mathrm{r}(\mathrm{C}-\mathrm{O}) / \AA$ & 1.230 & 1.231 & 1.233 & 1.231 \\
\hline $\mathrm{r}\left(\mathrm{C}-\mathrm{N}_{3}\right) / \AA$ & 1.394 & 1.389 & 1.380 & 1.381 \\
\hline $\mathrm{r}\left(\mathrm{C}-\mathrm{N}_{4}\right) / \AA$ & 1.394 & 1.389 & 1.380 & 1.395 \\
\hline$\theta\left(\mathrm{OCN}_{3}\right)$ & 123.3 & 122.6 & 122.6 & 122.7 \\
\hline$\theta\left(\mathrm{OCN}_{4}\right)$ & 123.3 & 122.6 & 122.6 & 122.7 \\
\hline$\tau\left(\mathrm{OCN}_{3} \mathrm{~N}_{4}\right)$ & 180.0 & 174.7 & 180.0 & 175.5 \\
\hline $\mathrm{r}\left(\mathrm{N}_{3} \mathrm{H}_{5}\right) / \AA$ & 1.014 & 1.012 & 1.008 & 1.009 \\
\hline $\mathrm{r}\left(\mathrm{N}_{3} \mathrm{H}_{6}\right) / \AA$ & 1.014 & 1.012 & 1.009 & 1.010 \\
\hline$\theta\left(\mathrm{CN}_{3} \mathrm{H}_{5}\right)$ & 116.5 & 119.8 & 123.5 & 122.3 \\
\hline$\theta\left(\mathrm{CN}_{3} \mathrm{H}_{6}\right)$ & 112.5 & 113.9 & 117.0 & 116.4 \\
\hline$\tau\left(\mathrm{OCN}_{3} \mathrm{H}_{5}\right)$ & 148.3 & -159.9 & 180.0 & -172.0 \\
\hline$\tau\left(\mathrm{OCN}_{3} \mathrm{H}_{6}\right)$ & 13.9 & -16.6 & 0.0 & -11.7 \\
\hline $\mathrm{r}\left(\mathrm{N}_{4} \mathrm{H}_{7}\right) / \AA$ & 1.014 & 1.012 & 1.008 & 1.013 \\
\hline $\mathrm{r}\left(\mathrm{N}_{4} \mathrm{H}_{8}\right) / \AA$ & 1.014 & 1.012 & 1.009 & 1.014 \\
\hline$\theta\left(\mathrm{OCN}_{4} \mathrm{H}_{7}\right)$ & 116.5 & 119.8 & 123.5 & 118.3 \\
\hline$\theta\left(\mathrm{OCN}_{4} \mathrm{H}_{8}\right)$ & 112.5 & 113.9 & 117.0 & 112.6 \\
\hline$\tau\left(\mathrm{OCN}_{4} \mathrm{H}_{7}\right)$ & 148.3 & 159.9 & 180.0 & 153.3 \\
\hline$\tau\left(\mathrm{OCN}_{4} \mathrm{H}_{8}\right)$ & 13.9 & 16.6 & 0.0 & 16.1 \\
\hline
\end{tabular}

Table 3.1: Important conformations in inversion PES of urea molecule 


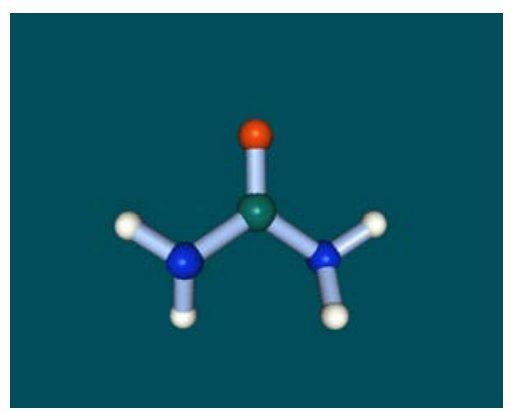

Figure 3.2: A - global minimum, $\mathrm{C}_{2}$

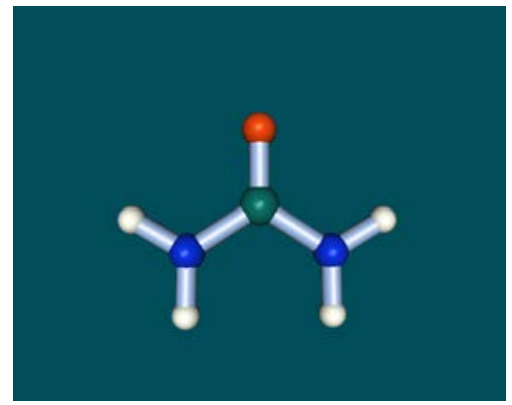

Figure 3.4: $\mathrm{C}$ - rank-2 saddle, $\mathrm{C}_{2 v}$

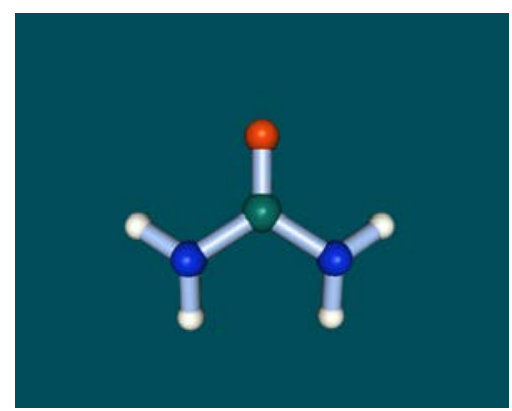

Figure 3.3: $\mathrm{B}$ - local minimum, $\mathrm{C}_{s}$

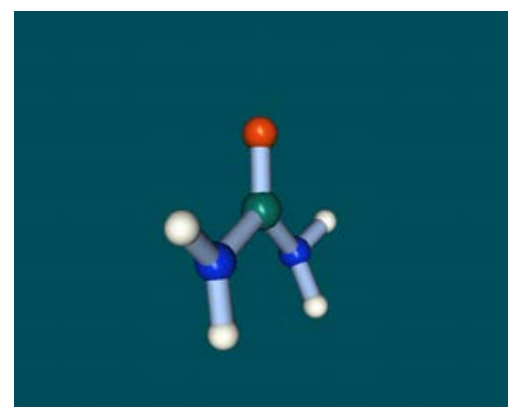

Figure 3.5: D - rank-1 saddle, $\mathrm{C}_{1}$

"out-of-plane" angle measurement. The out-of-plane angle is 31.5 for a perfect $s p^{3}$ hybridization and 0 for $s p^{2}$ hybridization. As an example, ammonia molecule's out-ofplane angle is 38.5 .

\begin{tabular}{|c|c|c|}
\hline & out-of-plane angle 1 & out-of-plane angle 2 \\
\hline Conformation A & 17.0 & 17.0 \\
\hline Conformation B & 10.6 & 10.6 \\
\hline Conformation C & 0.0 & 0.0 \\
\hline Conformation D & 3.0 & 14.6 \\
\hline
\end{tabular}

Table 3.2: Out-of plane angle of different urea molecule conformations in its inversion PES

The rank-1 saddle (conformation D) connects global and local energy minimum (conformation A and B). From its out-of-plane angles (Table 3.2) we can tell this connection is through inverting only one trigonal pyramid structure of $-\mathrm{NH}_{2}$ group: one of the two nitrogen atoms in this conformation is very close to $s p^{2}$ hybridization (out-of-plane angle is 3.0), which makes the conformation half-side planar. 


\subsection{Inversion PES}

\subsubsection{Inversion Barrier}

Normal coordinate calculations using infrared and Raman spectra data for solid urea give an estimated nitrogen inversion barrier of $1.29 \mathrm{kcal} / \mathrm{mol}$ [49].

As PES shown (Fig. 3.1), the lowest energy barrier for inversion is merely 1.09 $\mathrm{kcal} / \mathrm{mol}$, which takes the path through conformation B and D. One might compare this barrier to a typical inversion energy barrier of ammonia molecule, which is about 2.5 $\mathrm{kcal} / \mathrm{mol}$. Even the higher inversion barrier, which takes the path through conformation $\mathrm{C}$, is only $1.58 \mathrm{kcal} / \mathrm{mol}$. All these make urea molecule's inversion PES a fairly flat one, and the urea molecule can easily switch itself between planar and nonplanar structures.

There exist three paths connecting the two global energy minima as the PES shown (Fig. 3.1). Since all these three energy barriers are very low, the three paths are all important and have to be included while one carries out dynamic calculations. This feature makes urea molecule a very interesting dynamic system as an inversion model.

\subsubsection{Population Map}

Population map were generated from the inversion PES data. First, at each data point a probability $Z_{o}$ was calculated:

$$
Z_{o}=\exp \left[-E\left(\tau_{1}^{\prime}, \tau_{2}^{\prime}\right) / k_{B} T\right]
$$

where

$$
\tau_{1}^{\prime}=\tau_{5}-\tau_{6} \quad \text { and } \quad \tau_{2}^{\prime}=\tau_{7}-\tau_{8}
$$

The probability function then integrated numerically using Simpson'rule [50] over the surface range of $[120,240] \times[120,240]$ to produce the normalization factor $Z_{n}$ :

$$
Z_{n}=\int_{120}^{240} \int_{120}^{240} \exp \left[-E\left(\tau_{1}^{\prime}, \tau_{2}^{\prime}\right) / k_{B} T\right] \mathrm{d} \tau_{1}^{\prime} \mathrm{d} \tau_{2}^{\prime}
$$


Finally after normalizing the probability $Z_{o}$,

$$
Z_{n}=\frac{Z_{o}}{Z_{n}}
$$

a probability map was generated (Fig. 3.6), with contours defining the region of conformational space containing defined percentage of molecules.

It is clear from the plot that urea is a very flexible molecule. Only 50 per cent molecules take the conformations around the global energy minimum. Another half is off from the most stable conformation.

\subsubsection{Dipole Moment}

The dipole moment of urea measured is $4.56 \mathrm{D}$ in a non-polar solvent (dioxane) 20, $4.2 \mathrm{D}$ in water, $6.25 \mathrm{D}$ in acetone, 51 , and $3.83 \mathrm{D}$ in gas states 31 .

My calculations show that the dipole moment is 4.31D for the planar structure and 3.57D for the global minimum conformation (Table 3.3.

\begin{tabular}{|l|c|c|c|}
\hline & CO bond length / & out of plane angle & dipole moment /D \\
\hline global minimum & 1.2296 & 17.0 & 3.57 \\
\hline planar structure & 1.2328 & 0.0 & 4.31 \\
\hline
\end{tabular}

Table 3.3: Dipole moment of urea molecules

It is easy to understand why the planar structure is more polar. Urea molecules can be described as a combination of two mesomeric structures (Fig. 3.7) due to resonance. The CO bond of carbonyl group in structure (B) and (C) is longer than that in structure (A) due to their single and double bonding nature (Table 3.3). Also, the oxygen atom carries a larger negative charge than that in structure (A). Both effects make structure (B) and (C) a higher dipole moment than that in structure (A).

The resonance is easier to happen while the nitrogen atom has more $s p^{2}$ hybridization than $s p^{3}$, which is the case of planar structure (Table 3.3 , the planar structure 


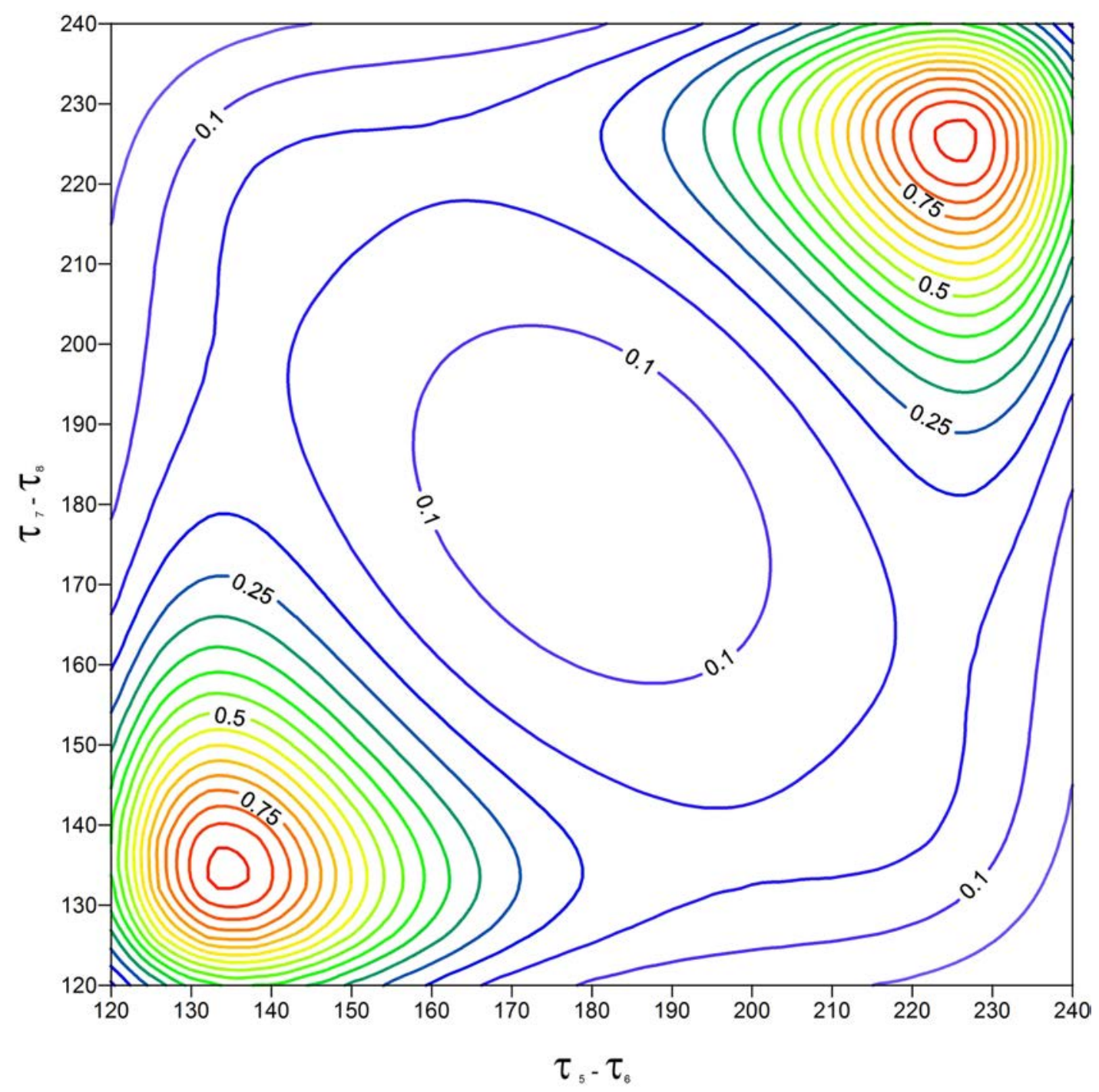

Figure 3.6: Population map - corresponding to inversion PES of urea molecule at $37^{\circ} \mathrm{C}$. 
has a smaller out of plane angle). Therefore, the planar structure favors structure (B) and $(\mathrm{C})$ more than that in global energy minimum structure and has a higher dipole moment.<smiles>NC(=[NH2+])CCCCC(N)=O</smiles>

Figure 3.7: Mesomeric structures of urea molecule - adapted from figure 2, Keuleers, R. \& Desseyn, H. O., Journal of Physical Chemistry A, 1999, 103, 4621-4630.

Hydrogen bonding to carbonyl group always weakens the CO bond, therefore makes its length longer and increases the dipole moment. It is not surprised that urea molecules have a higher dipole moment in solution than that in gas states due to the interaction between them and solvent molecules. 


\subsection{Fitting inversion PES}

Inversion PES (Fig. 3.1) was plotted using variables $\tau_{1}$ and $\tau_{2}$ (section 2.3). They are the differences between two dihedral angles which are associated with hydrogen atoms in the same amino group. Since all the conformations on the inversion PES are not far away from conformation D (planar structure), the geometry of amino groups are fairly flat. One of these two dihedral angles are always close to 0 and another close to $\pi$, and their difference is close to $\pi$. In order to use variables close to 0 to fit the PES, new invariables were introduced:

$$
\varphi_{1}=\tau_{1}-\pi=\left(\tau_{5}-\tau_{6}\right)-\pi, \quad \varphi_{2}=\tau_{2}-\pi=\left(\tau_{7}-\tau_{8}\right)-\pi
$$

PES then was fitted using new variables $\varphi_{1}$ and $\varphi_{2}$ :

$$
V\left(\varphi_{1}, \varphi_{2}\right)=\sum_{a+b \leq 8} f_{a b}\left(\varphi_{1}\right)^{a}\left(\varphi_{2}\right)^{b}
$$

Totally 45 terms were generated but only 15 coefficients are independent due to symmetry restriction:

$$
f_{a b}=0 \text { when }(\mathrm{a}+\mathrm{b}) \text { is odd, }
$$

and

$$
f_{a b}=f_{b a} .
$$

All independent coefficients are listed in Table 3.4 (units in $\left.\mathrm{cm}^{-1}\right)$. Root mean square error is 1.664379332, and coefficient of determination $\left(R^{2}\right)$ is 0.999999715 .

The constant term $f_{00}=551.2 \mathrm{~cm}^{-1}$ is the energy of planar structure (conformation C). 


\begin{tabular}{|r|r|r|r|}
\hline$f_{00}$ & 551.2 & $f_{20}$ & -569.77 \\
$f_{11}$ & -362.15 & $f_{40}$ & 184.78 \\
$f_{31}$ & -6.52 & $f_{22}$ & 218.35 \\
$f_{60}$ & 297.04 & $f_{51}$ & 41.7 \\
$f_{42}$ & 29.77 & $f_{33}$ & -8.9 \\
$f_{80}$ & -30.98 & $f_{71}$ & -3.47 \\
$f_{62}$ & -16.57 & $f_{53}$ & -4.68 \\
$f_{44}$ & 8.44 & & \\
\hline
\end{tabular}

Table 3.4: Coefficients of fitted inversion PES of urea - units in $\mathrm{cm}^{-1}$ 


\subsection{Normal Mode Analysis}

\subsubsection{Degrees of Freedom}

A urea molecule has eight atoms. By removing three translational degrees of freedom and three rotational degrees of freedom, we have $(3 \times 8-6=18)$ degrees of freedom left. Any internal coordinate system to describe urea molecule should have 18 coordinates. In this study, the following 18 internal coordinates were used (atom numbering refers to Fig. 2.1):

\begin{tabular}{|c|c|}
\hline $\mathrm{r}(\mathrm{C}-\mathrm{O})$ & bond length of $\mathrm{C}_{1} \mathrm{O}_{2}$ bond \\
\hline $\mathrm{r}\left(\mathrm{C}_{-} \mathrm{N}_{3}\right)$ & bond length of $\mathrm{C}_{1} \mathrm{~N}_{3}$ bond \\
\hline $\mathrm{r}\left(\mathrm{C}_{-} \mathrm{N}_{4}\right)$ & bond length of $\mathrm{C}_{1} \mathrm{~N}_{4}$ bond \\
\hline$\theta\left(\mathrm{OCN}_{3}\right)$ & bond angle between $\mathrm{C}_{1} \mathrm{O}_{2}$ bond and $\mathrm{C}_{1} \mathrm{~N}_{3}$ bond \\
\hline$\theta\left(\mathrm{OCN}_{4}\right)$ & bond angle between $\mathrm{C}_{1} \mathrm{O}_{2}$ bond and $\mathrm{C}_{1} \mathrm{~N}_{4}$ bond \\
\hline$\tau\left(\mathrm{OCN}_{3} \mathrm{~N}_{4}\right)$ & dihedral angle between $\mathrm{O}_{2} \mathrm{C}_{1} \mathrm{~N}_{3}$ plane and $\mathrm{C}_{1} \mathrm{~N}_{3} \mathrm{~N}_{4}$ plane \\
\hline $\mathrm{r}_{3}\left(\mathrm{~N}_{3} \mathrm{H}_{5}\right)$ & bond length of $\mathrm{N}_{3} \mathrm{H}_{5}$ bond \\
\hline $\mathrm{r}\left(\mathrm{N}_{3} \mathrm{H}_{6}\right)$ & bond length of $\mathrm{N}_{3} \mathrm{H}_{6}$ bond \\
\hline$\theta\left(\mathrm{CN}_{3} \mathrm{H}_{5}\right)$ & bond angle between $\mathrm{C}_{1} \mathrm{~N}_{3}$ bond and $\mathrm{N}_{3} \mathrm{H}_{5}$ bond \\
\hline$\theta\left(\mathrm{CN}_{3} \mathrm{H}_{6}\right)$ & bond angle between $\mathrm{C}_{1} \mathrm{~N}_{3}$ bond and $\mathrm{N}_{3} \mathrm{H}_{6}$ bond \\
\hline$\tau\left(\mathrm{OCN}_{3} \mathrm{H}_{5}\right)$ & dihedral angle between $\mathrm{O}_{2} \mathrm{C}_{1} \mathrm{~N}_{3}$ plane and $\mathrm{C}_{1} \mathrm{~N}_{3} \mathrm{H}_{6}$ plane \\
\hline$\tau\left(\mathrm{OCN}_{3} \mathrm{H}_{6}\right)$ & dihedral angle between $\mathrm{O}_{2} \mathrm{C}_{1} \mathrm{~N}_{3}$ plane and $\mathrm{C}_{1} \mathrm{~N}_{3} \mathrm{H}_{6}$ plane \\
\hline $\mathrm{r}\left(\mathrm{N}_{4} \mathrm{H}_{7}\right)$ & bond length of $\mathrm{N}_{4} \mathrm{H}_{7}$ bond \\
\hline $\mathrm{r}\left(\mathrm{N}_{4} \mathrm{H}_{8}\right)$ & bond length of $\mathrm{N}_{4} \mathrm{H}_{8}$ bond \\
\hline$\theta\left(\mathrm{CN}_{4} \mathrm{H}_{7}\right)$ & bond angle between $\mathrm{C}_{1} \mathrm{~N}_{4}$ bond and $\mathrm{N}_{4} \mathrm{H}_{7}$ bond \\
\hline$\theta\left(\mathrm{CN}_{4} \mathrm{H}_{8}\right)$ & bond angle between $\mathrm{C}_{1} \mathrm{~N}_{4}$ bond and $\mathrm{N}_{4} \mathrm{H}_{8}$ bond \\
\hline$\tau\left(\mathrm{OCN}_{4} \mathrm{H}_{7}\right)$ & dihedral angle between $\mathrm{O}_{2} \mathrm{C}_{1} \mathrm{~N}_{4}$ plane and $\mathrm{C}_{1} \mathrm{~N}_{4} \mathrm{H}_{7}$ plane \\
\hline$\tau\left(\mathrm{OCN}_{4} \mathrm{H}_{8}\right)$ & dihedral angle between $\mathrm{O}_{2} \mathrm{C}_{1} \mathrm{~N}_{4}$ plane and $\mathrm{C}_{1} \mathrm{~N}_{4} \mathrm{H}_{8}$ plane \\
\hline
\end{tabular}

Table 3.5: Conventional internal coordinates of urea molecule

\subsubsection{Kinetic Energy Terms}

The kinetic energy terms transformed from conventional internal coordinates to any internal coordinates can be derived from the fundamental formula:

$$
2 T=P^{\prime} G P
$$


where $T$ is the kinetic energy, $P$ is a column vector whose elements are the momenta conjugate to the a given set of internal coordinate $Q$ 's. Since there are always 18 internal coordinates, $P$ is a column vector with 18 components.

$G$ is the kinetic energy matrix whose elements were given by Decius [52],

$$
g_{q q^{\prime}}=\sum_{k=1}^{N} S_{q k} \cdot S_{q^{\prime} k} \mu_{k}
$$

in which $q$ and $q^{\prime}$ are conventional internal coordinates (for urea molecule, they are described in Tab. 3.5), $\mu_{k}$ is the reciprocal mass of the $k$ th atom, and $S_{q k}$ is a vector which represents the contribution of the $k$ th atom to $q$. The matrix elements $g_{q q^{\prime}}$ represents the interaction between momenta associated with coordinates $q$ and $q^{\prime}$. There are total 33 types of $g_{q q^{\prime}}$ 's and their general formulas were listed by Decius [52].

For urea molecule, the changes in most internal coordinates are very small during the molecular conformation switching processes and infrared spectra did show that they are not coupled with the motion of amino groups [43]. Therefore, it is reasonable only to take the four dihedral angles involving hydrogen atoms in amino groups as explicit variables. All other conventional internal coordinates were treated as fixed parameters by taking the value at their optima conformation to reduce the dimensions of the problem.

$$
2 T=\left(P_{\tau_{1}}, P_{\tau_{2}}, P_{\tau_{3}}, P_{\tau_{4}}\right)\left(\begin{array}{cccc}
g_{11} & g_{12} & g_{13} & g_{14} \\
g_{21} & g_{22} & g_{23} & g_{24} \\
g_{31} & g_{32} & g_{33} & g_{34} \\
g_{41} & g_{42} & g_{43} & g_{44}
\end{array}\right)\left(\begin{array}{c}
P_{\tau_{1}} \\
P_{\tau_{2}} \\
P_{\tau_{3}} \\
P_{\tau_{4}}
\end{array}\right)
$$

Here,

$$
\tau_{1}=\tau\left(\mathrm{OCN}_{3} \mathrm{H}_{5}\right), \quad \tau_{2}=\tau\left(\mathrm{OCN}_{3} \mathrm{H}_{6}\right), \quad \tau_{3}=\tau\left(\mathrm{OCN}_{4} \mathrm{H}_{7}\right), \quad \tau_{4}=\tau\left(\mathrm{OCN}_{4} \mathrm{H}_{8}\right) .
$$


All the $G$ matrix elements were calculated using equation 4.4, and internal coordinate values used have been listed in Table 3.1. Calculated $G$ matrix elements are listed as below (units in $\mathrm{cm}^{-1}$ ):

$$
\begin{aligned}
& g_{11}=2176.19+247.57 * \cos \tau_{1} \\
& g_{22}=2473.13+298.94 * \cos \tau_{2} \\
& g_{33}=2176.19+247.57 * \cos \tau_{3} \\
& g_{44}=2473.13+298.94 * \cos \tau_{4} \\
& g_{12}=324.10+123.78 * \cos \tau_{1}+149.47 * \cos \tau_{2}+254.49 * \cos \left(\tau_{1}-\tau_{2}\right) \\
& g_{21}=324.10+123.78 * \cos \tau_{1}+149.47 * \cos \tau_{2}+254.49 * \cos \left(\tau_{1}-\tau_{2}\right) \\
& g_{34}=324.10+123.78 * \cos \tau_{3}+149.47 * \cos \tau_{4}+254.49 * \cos \left(\tau_{3}-\tau_{4}\right) \\
& g_{43}=324.10+123.78 * \cos \tau_{3}+149.47 * \cos \tau_{4}+254.49 * \cos \left(\tau_{3}-\tau_{4}\right) \\
& g_{13}=-303.73-58.56 *\left(\cos \tau_{1}+\cos \tau_{3}\right)-10.78 * \cos \left(\tau_{1}+\tau_{3}\right)-4.39 * \cos \left(\tau_{1}-\tau_{3}\right) \\
& g_{31}=-303.73-58.56 *\left(\cos \tau_{1}+\cos \tau_{3}\right)-10.78 * \cos \left(\tau_{1}+\tau_{3}\right)-4.39 * \cos \left(\tau_{1}-\tau_{3}\right) \\
& g_{24}=-303.73-75.87 *\left(\cos \tau_{2}+\cos \tau_{4}\right)-18.10 * \cos \left(\tau_{2}+\tau_{4}\right)-7.45 * \cos \left(\tau_{2}-\tau_{4}\right) \\
& g_{42}=-303.73-75.87 *\left(\cos \tau_{2}+\cos \tau_{4}\right)-18.10 * \cos \left(\tau_{2}+\tau_{4}\right)-7.45 * \cos \left(\tau_{2}-\tau_{4}\right) \\
& g_{14}=-303.73-58.56 * \cos \tau_{1}-75.87 * \cos \tau_{4}-13.98 * \cos \left(\tau_{1}+\tau_{4}\right)-5.72 * \cos \left(\tau_{1}-\tau_{4}\right) \\
& g_{41}=-303.73-58.56 * \cos \tau_{1}-75.87 * \cos \tau_{4}-13.98 * \cos \left(\tau_{1}+\tau_{4}\right)-5.72 * \cos \left(\tau_{1}-\tau_{4}\right) \\
& g_{23}=-303.73-58.56 * \cos \tau_{2}-75.87 * \cos \tau_{3}-13.98 * \cos \left(\tau_{2}+\tau_{3}\right)-5.72 * \cos \left(\tau_{2}-\tau_{3}\right) \\
& g_{32}=-303.73-58.56 * \cos \tau_{2}-75.87 * \cos \tau_{3}-13.98 * \cos \left(\tau_{2}+\tau_{3}\right)-5.72 * \cos \left(\tau_{2}-\tau_{3}\right)
\end{aligned}
$$

\subsubsection{Potential Energy Terms}

For all important conformations located in inversion PES (conformation A, B, C, and D in Fig. 3.1 , $\tau_{1}$ and $\tau_{3}$ are close to 0 and $\tau_{2}$ and $\tau_{4}$ are close to $\pi$. In order to expand 
energy terms using variables close to 0 , the following transformation were applied:

$$
\tau_{2}^{\prime}=\tau_{2}-\pi, \quad \tau_{4}^{\prime}=\tau_{4}-\pi
$$

It is trivial that under this transformation we have:

$$
P_{\tau_{2}^{\prime}}=P_{\tau_{2}}, \quad P_{\tau_{4}^{\prime}}=P_{\tau_{4}}
$$

but many terms in $g$ will change their signs:

$$
\cos \tau_{2}^{\prime}=-\cos \tau_{2}, \quad \cos \tau_{4}^{\prime}=-\cos \tau_{4}
$$

The potential energy $V$ is then expressed as internal coordinates and the force constants matrix $F$ :

$$
2 V=\left(\tau_{1}, \tau_{2}^{\prime}, \tau_{3}, \tau_{4}^{\prime}\right)\left(\begin{array}{cccc}
f_{11} & f_{12} & f_{13} & f_{14} \\
f_{21} & f_{22} & f_{23} & f_{24} \\
f_{31} & f_{32} & f_{33} & f_{34} \\
f_{41} & f_{42} & f_{43} & f_{44}
\end{array}\right)\left(\begin{array}{c}
\tau_{1} \\
\tau_{2}^{\prime} \\
\tau_{3} \\
\tau_{4}^{\prime}
\end{array}\right)
$$

Elements of matrix $F$ can be found by first fitting energy $V$ into a polynomial of $\tau_{1}$, $\tau_{2}^{\prime}, \tau_{3}$ and $\tau_{4}^{\prime}$ :

$$
V=\sum f_{a b c d}\left(\tau_{1}\right)^{a}\left(\tau_{2}^{\prime}\right)^{b}\left(\tau_{3}\right)^{c}\left(\tau_{4}^{\prime}\right)^{d}
$$


then taken from coefficients of all quadratic terms:

$$
\begin{array}{ll}
f_{11}=f_{2000} \times 2 & f_{22}=f_{0200} \times 2 \\
f_{33}=f_{0020} \times 2 & f_{44}=f_{0002} \times 2 \\
f_{12}=f_{1100} & f_{21}=f_{1100} \\
f_{13}=f_{1010} & f_{31}=f_{1010} \\
f_{14}=f_{1001} & f_{41}=f_{1001} \\
f_{23}=f_{0110} & f_{32}=f_{0110} \\
f_{24}=f_{0101} & f_{42}=f_{0101} \\
f_{34}=f_{0011} & f_{43}=f_{0011}
\end{array}
$$

\subsubsection{Normal mode coordinates}

By solving secular equation

$$
|G F-E \lambda|=0
$$

eigenvalues and corresponding eigenvectors can be obtained. Let $L$ be the matrix which its column vectors are the eigenvectors of matrix $G F, q$ be the column vector of conventional internal coordinates, then the transformation:

$$
Q=L^{-1} q
$$

will generate the normal mode coordinates $Q$.

In normal mode coordinates, matrix $G$ and $F$ have simple forms:

$$
L^{\prime} G^{-1} L=E, \quad L^{\prime} F L=\left(\begin{array}{ccc}
\lambda_{1} & & \\
& \lambda_{2} & \\
& & \ldots
\end{array}\right)
$$




\subsubsection{Rank-2 Saddle}

At the rank-2 saddle point conformation (conformation C in Fig. 3.1), we have:

$$
\tau_{1}=0, \quad \tau_{2}^{\prime}=0\left(\tau_{2}=\pi\right), \quad \tau_{3}=0, \quad \tau_{4}^{\prime}=0\left(\tau_{4}=\pi\right)
$$

The $G$ matrix at the rank-2 saddle point can be calculated by substituting the above values for each $g$ element (units in $\mathrm{cm}^{-1}$ ):

$$
G=\left(\begin{array}{rrrr}
2423.75 & 43.92 & -436.04 & -266.73 \\
43.92 & 2174.19 & -301.34 & -177.56 \\
-436.04 & -301.34 & 2423.75 & 43.92 \\
-266.73 & -177.56 & 43.92 & 2174.19
\end{array}\right)
$$

From all these off-diagonal elements, it is clear that the inversions of two $-\mathrm{NH}_{2}$ amino groups are coupled together, which is in accord with infrared spectra observation [53].

Around the rank-2 saddle points, 1800 conformations were calculated randomly in the range of $\tau_{1}= \pm 5, \tau_{2}^{\prime}= \pm 5, \tau_{3}= \pm 5, \tau_{4}^{\prime}= \pm 5$, and the $F$ matrix is found by fitting $V$ into the following formula (see Appendix A for details):

$$
V=\sum_{a+b+c+d \leq 4} f_{a b c d}\left(\tau_{1}\right)^{a}\left(\tau_{2}^{\prime}\right)^{b}\left(\tau_{3}\right)^{c}\left(\tau_{4}^{\prime}\right)^{d}
$$

The $F$ matrix is found to be (units in $\mathrm{cm}^{-1}$ ):

$$
F=\left(\begin{array}{rrrr}
2967.14 & 3248.45 & -1664.66 & -704.73 \\
3248.45 & 258.87 & -704.73 & -1399.96 \\
-1664.66 & -704.73 & 2967.14 & 3248.45 \\
-704.73 & -1399.96 & 3248.45 & 258.87
\end{array}\right)
$$

Secular equation $|G F-E \lambda|=0$ was solved to find out the transformation which leads 
to normal mode coordinates:

$$
\begin{aligned}
& \phi_{1}=0.01057 *\left(\tau_{1}-\tau_{3}\right)+0.00740 *\left(\tau_{2}^{\prime}-\tau_{4}^{\prime}\right) \\
& \phi_{2}=-0.00769 *\left(\tau_{1}+\tau_{3}\right)+0.01295 *\left(\tau_{2}^{\prime}+\tau_{4}^{\prime}\right) \\
& \phi_{3}=0.01401 *\left(\tau_{1}+\tau_{3}\right)+0.00930 *\left(\tau_{2}^{\prime}+\tau_{4}^{\prime}\right) \\
& \phi_{4}=0.00812 *\left(\tau_{1}-\tau_{3}\right)-0.01270 *\left(\tau_{2}^{\prime}-\tau_{4}^{\prime}\right)
\end{aligned}
$$

The kinetic energy and potential energy terms under the normal mode coordinates were found to be:

$$
\begin{gathered}
2 T=P_{\phi_{1}}^{2}+P_{\phi_{2}}^{2}+P_{\phi_{3}}^{2}+P_{\phi_{4}}^{2} \\
2 V=4 \pi^{2} \nu_{1}^{2} \phi_{1}^{2}-4 \pi^{2} \nu_{2}^{2} \phi_{2}^{2}+4 \pi^{2} \nu_{3}^{2} \phi_{3}^{2}-4 \pi^{2} \nu_{4}^{2} \phi_{4}^{2} \\
\nu_{1}=748.5 \mathrm{~cm}^{-1}, \quad \nu_{2}=391.5 \mathrm{~cm}^{-1}, \quad \nu_{3}=360.9 \mathrm{~cm}^{-1}, \quad \nu_{4}=245.4 \mathrm{~cm}^{-1}
\end{gathered}
$$

These low-lying vibration modes indicate that vibrations on urea molecule's inversional PES might be treated as large amplitude motion. 


\subsection{Two-dimensional Schrödinger Equation}

In several experimental studies [32, 33, 54], the local energy minimum conformations (conformation B in Fig. 3.1) were considered not a distinct conformation but part of a large amplitude motion of the global energy minimum conformations (conformation A). With the fitted inversional PES at hand, it is possible to solve two-dimensional Schrödinger equation of urea molecule to find out the eigenvalues of low-lying vibration modes. By comparing these eigenvalues with inversional energy barrier, the validity of the large amplitude vibration can be justified.

The two-dimensional Schrödinger equation of urea molecule's inversion motion can be written as:

$$
-\frac{\hbar^{2}}{2} \nabla \cdot\left(\begin{array}{cc}
g_{\varphi_{1} \varphi_{1}} & g_{\varphi_{1} \varphi_{2}} \\
g_{\varphi_{2} \varphi_{1}} & g_{\varphi_{2} \varphi_{2}}
\end{array}\right) \nabla \psi\left(\varphi_{1}, \varphi_{2}\right)+V\left(\varphi_{1}, \varphi_{2}\right) \psi\left(\varphi_{1}, \varphi_{2}\right)=E_{i} \psi\left(\varphi_{1}, \varphi_{2}\right)
$$

where $g_{\varphi_{i} \varphi_{j}}$ is the $G$ matrix elements which associate with momenta $P_{\varphi_{1}}$ and $P_{\varphi_{2}}$, $V\left(\varphi_{1}, \varphi_{2}\right)$ is the fitted inversion potential energy terms (equation 3.1).

Schrödinger equation 3.3 was solved using Matlab's Partial Differential Equation Toolbox. Four eigenvalues were found in the range $E_{i}<1000 \mathrm{~cm}^{-1}$. They are 501.9 $\mathrm{cm}^{-1}, 599.1 \mathrm{~cm}^{-1}, 783.5 \mathrm{~cm}^{-1}$, and $862.0 \mathrm{~cm}^{-1}$ respectively (Table 3.6). Their contour maps and wave functions were plotted in Fig. 3.8 - 3.15 .

\begin{tabular}{|c|c|c|c|c|}
\hline Level & ground & first excited & second excited & third excited \\
\hline Energy $/ \mathrm{cm}^{-1}$ & 501.9 & 599.1 & 783.5 & 862.0 \\
\hline
\end{tabular}

Table 3.6: Energy levels of urea molecule's inversion potential

The ground state's eigen energy $\left(501.9 \mathrm{~cm}^{-1}\right)$ is lower than energy of the planar conformation $\left(552.6 \mathrm{~cm}^{-1}\right)$, but already higher than energies of the local energy minimum conformation $\left(370.7 \mathrm{~cm}^{-1}\right)$ and rank-1 saddle conformation $\left(381.2 \mathrm{~cm}^{-1}\right)$. The first excited state's eigen energy $\left(599.1 \mathrm{~cm}^{-1}\right)$ is higher than energies of all important 


\subsection{Two-dimensional Schrödinger Equation}

conformations in the inversion PES. Therefore, all these important conformations can be easily reached by these vibration modes and it could be reasonable to treat them as a large amplitude motion. 


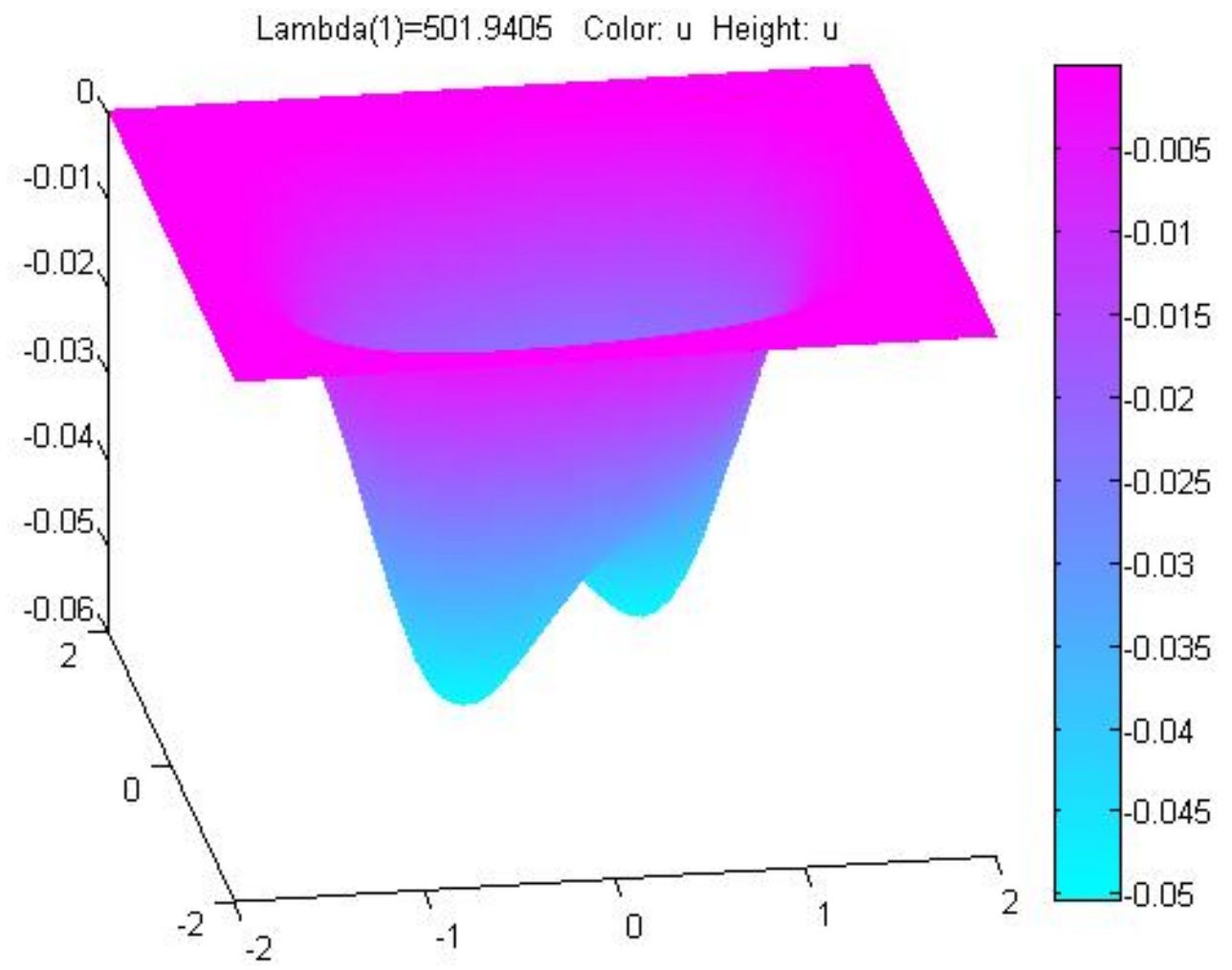

Figure 3.8: Ground state wave function of urea molecule from inversion potential - Eigenvalue $501.9 \mathrm{~cm}^{-1}$, plotted by Matlab.

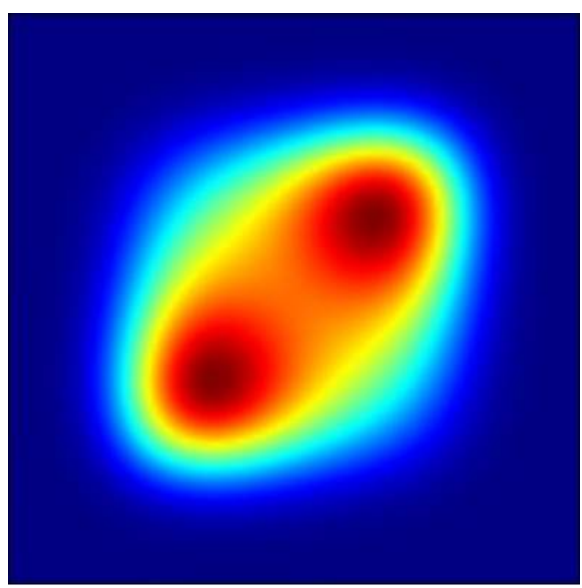

Figure 3.9: Contour map of ground state wave function of urea molecule from inversion potential - Eigenvalue $501.9 \mathrm{~cm}^{-1}$, plotted by Matlab. 


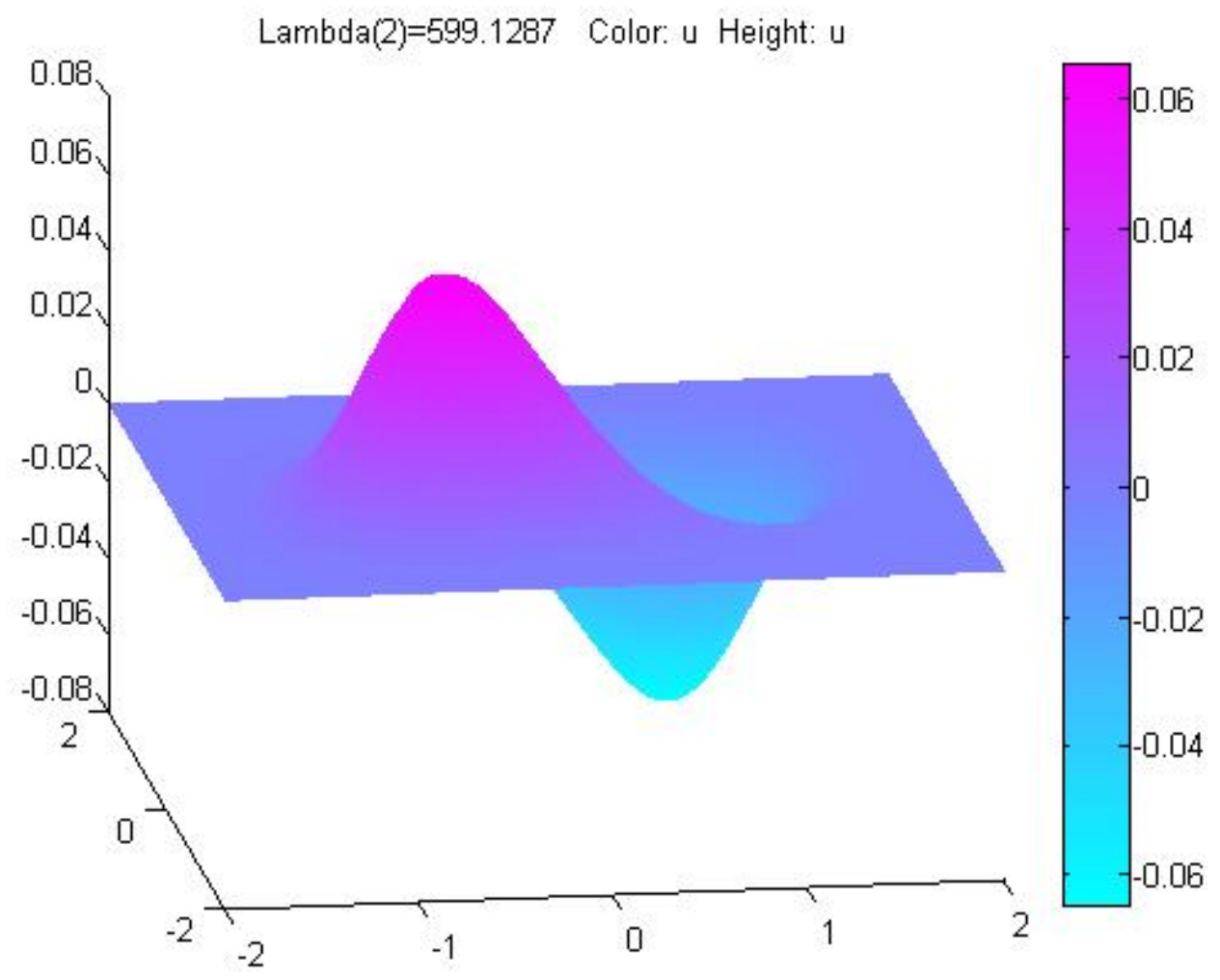

Figure 3.10: First excited state wave function of urea molecule from inversion potential - Eigenvalue $599.1 \mathrm{~cm}^{-1}$, plotted by Matlab.

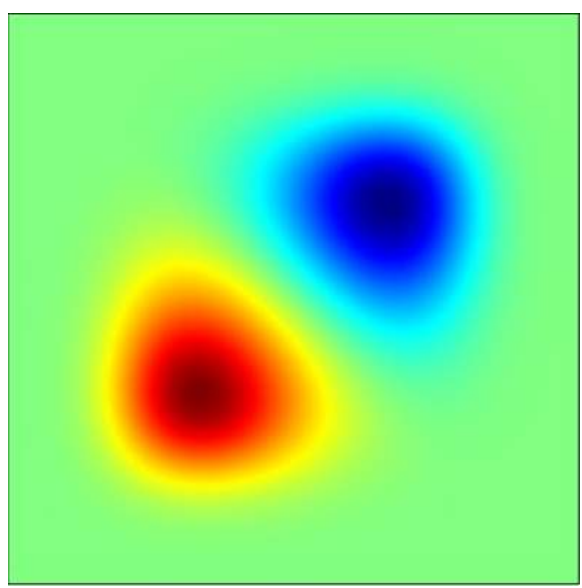

Figure 3.11: Contour map of first excited state wave function of urea molecule from inversion potential - Eigenvalue $599.1 \mathrm{~cm}^{-1}$, plotted by Matlab. 


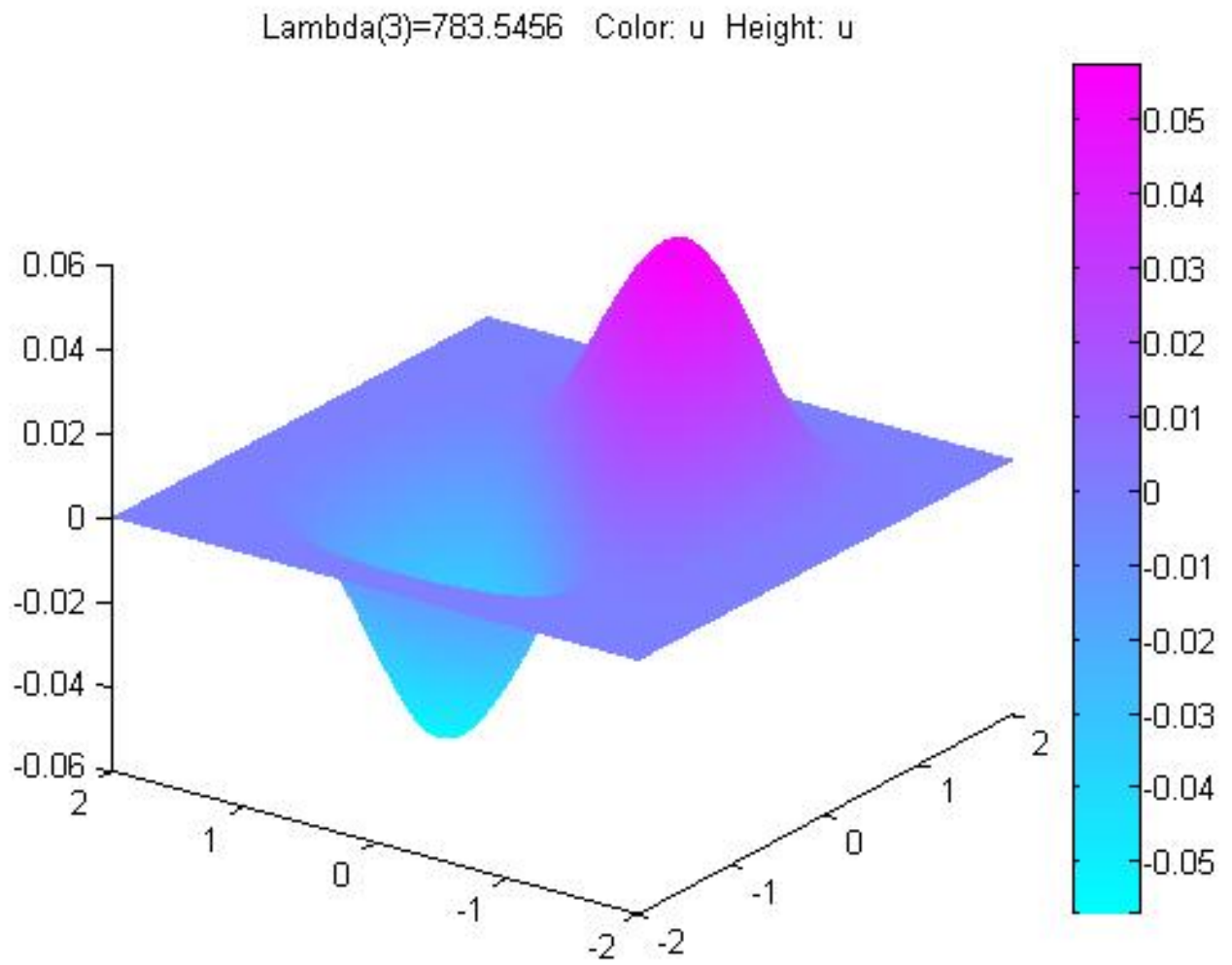

Figure 3.12: Second excited state wave function of urea molecule from inversion potential - Eigenvalue $783.5 \mathrm{~cm}^{-1}$, plotted by Matlab.

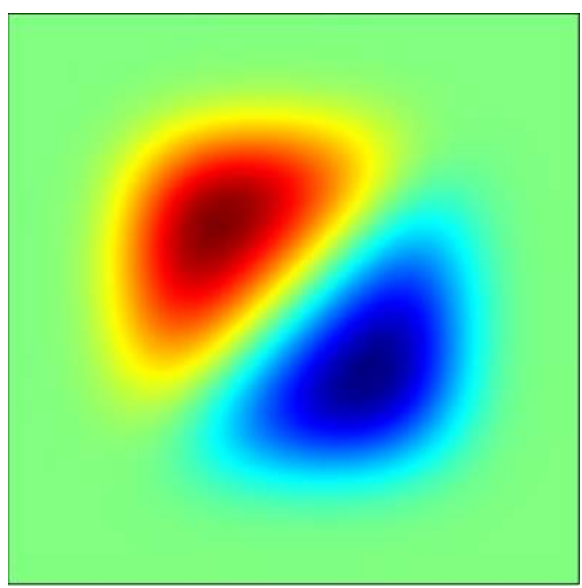

Figure 3.13: Contour map of second excited state wave function of urea molecule from inversion potential - Eigenvalue $783.5 \mathrm{~cm}^{-1}$, plotted by Matlab. 
Lambda(4)=861.96 Color: $u$ Height: $u$

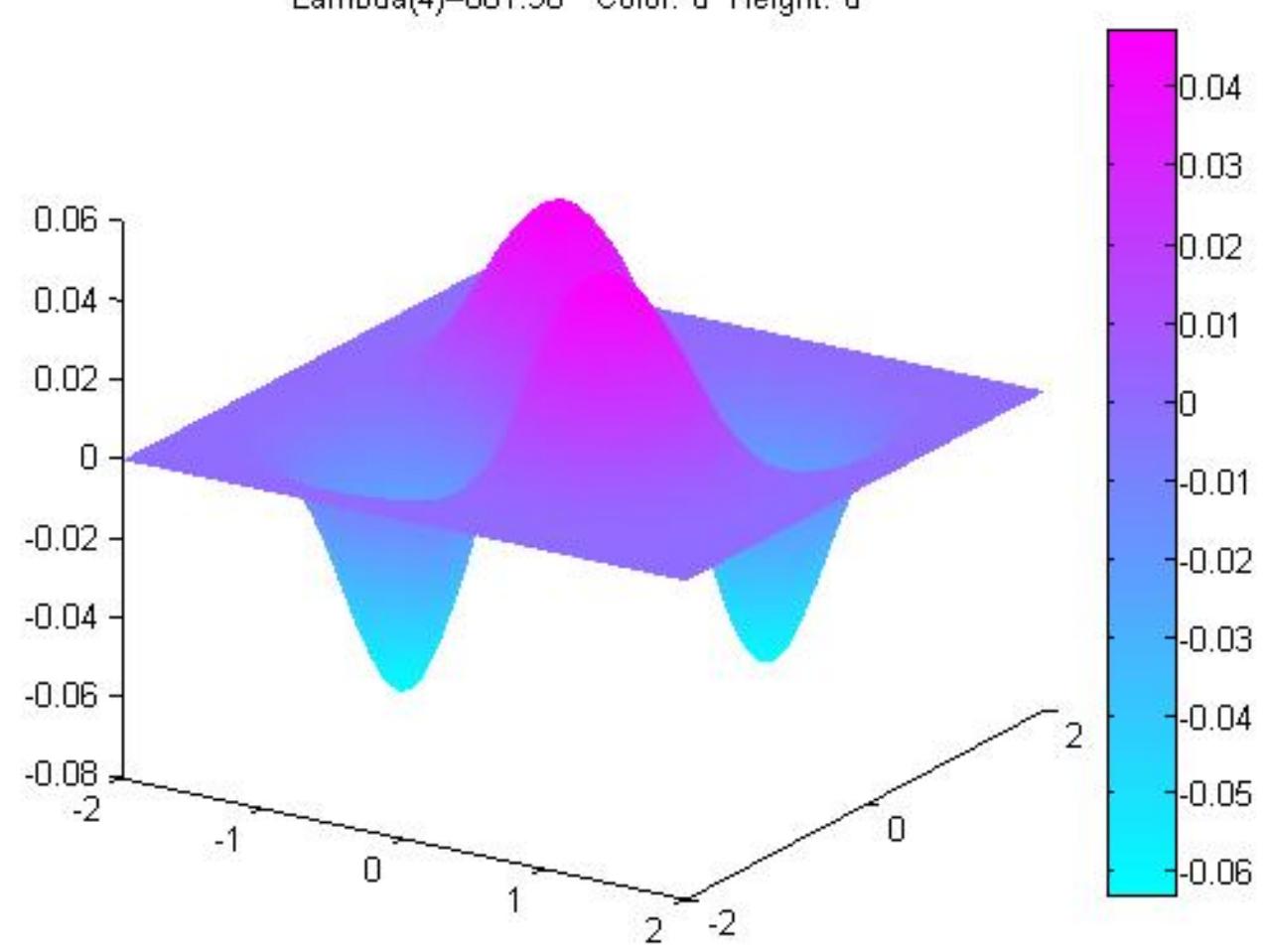

Figure 3.14: Third excited state wave function of urea molecule from inversion potential - Eigenvalue $862.0 \mathrm{~cm}^{-1}$, plotted by Matlab.

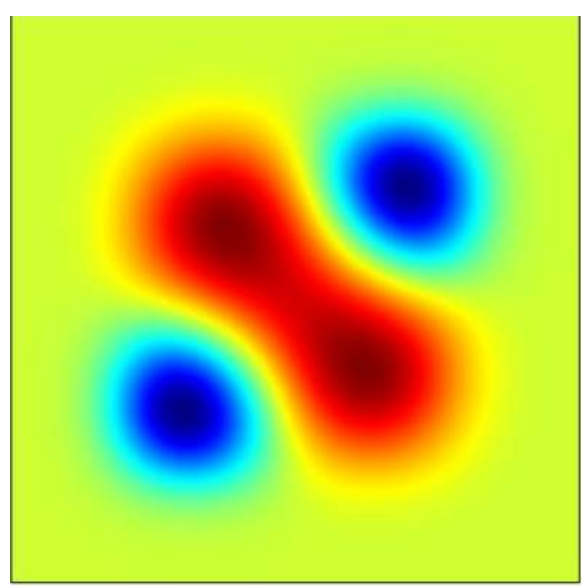

Figure 3.15: Contour map of third excited state wave function of urea molecule from inversion potential - Eigenvalue $862.0 \mathrm{~cm}^{-1}$, plotted by Matlab. 


\section{Chapter 4}

\section{Four-dimensional Rotation}

\section{Potential Energy Surface}

\subsection{Internal rotation PES}

Urea molecule's energy were calculated with two rotation variables $\theta_{1}$ and $\theta_{2}$ (described in section 2.3) changing from $0^{\circ}$ to $360^{\circ}$. At each point, the values of $\theta_{1}$ and $\theta_{2}$ were fixed, but all other 16 degrees of freedom of urea molecule were left relaxed. Urea molecule's geometry was then optimized and energy was calculated. Each energy generated a point in the PES. Totally 2088 points were calculated and a contour plot was done to represent the rotation PES of urea (Fig 4.1).

\subsection{Important conformations}

All important comforamtions on the rotation PES are labelled in Fig. 4.1 and showed in Fig. 4.2 - Fig. 4.6 ,

conformation A is a global energy minimum and conformation B is a local energy minimum. They have been discussed in section 3.1.1. 


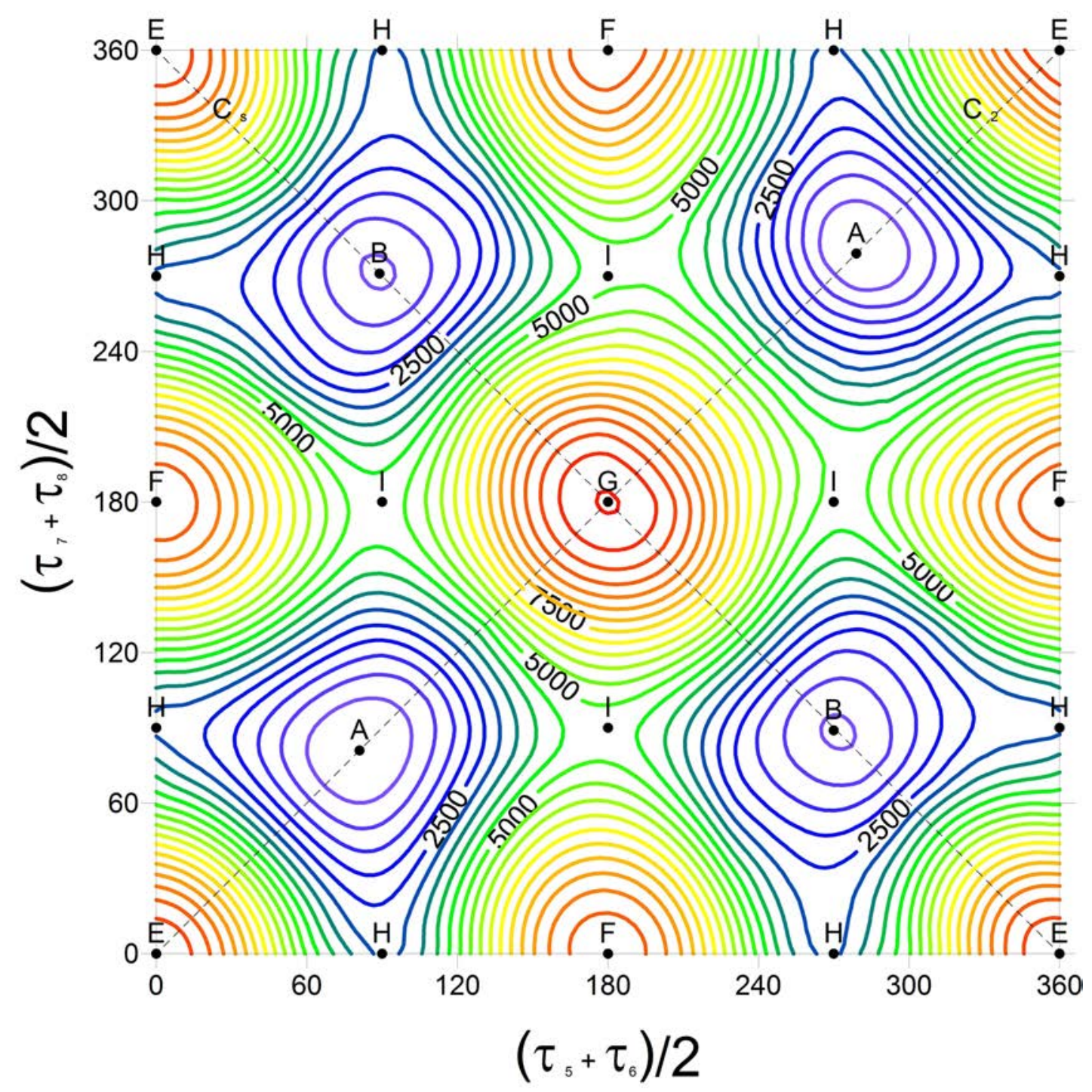

Figure 4.1: Rotational potential energy surface of urea molecule - unit in $\mathrm{cm}^{-1}$. 


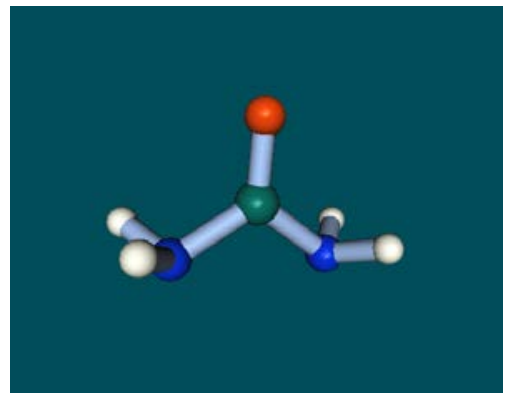

Figure 4.2: $\mathrm{E}$ - rank-2 saddle, $\mathrm{C}_{2 v}$

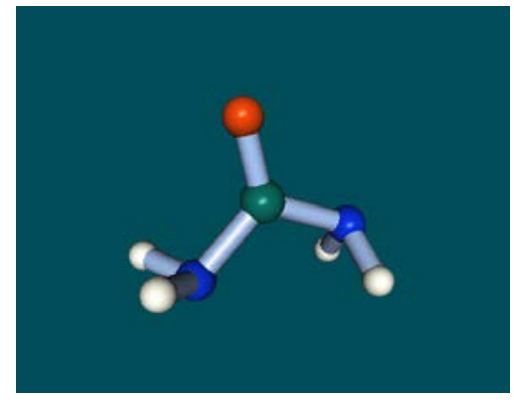

Figure 4.3: $\mathrm{F}$ - rank-2 saddle, $\mathrm{C}_{s}$

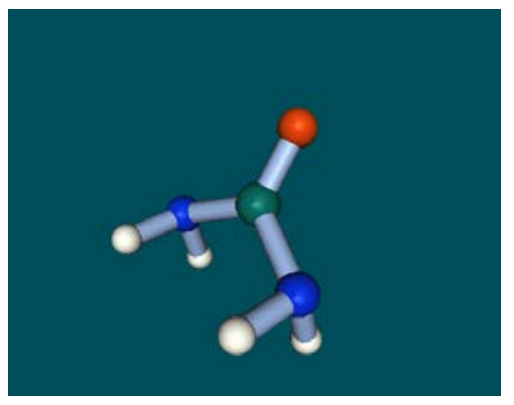

Figure 4.4: $\mathrm{G}$ - rank-2 saddle, $\mathrm{C}_{2 v}$

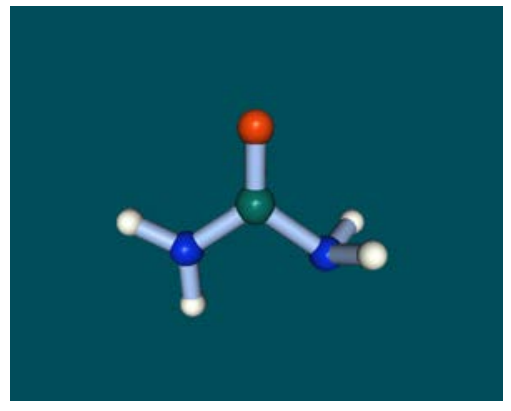

Figure 4.5: $\mathrm{H}$ - rank-1 saddle, $\mathrm{C}_{s}$

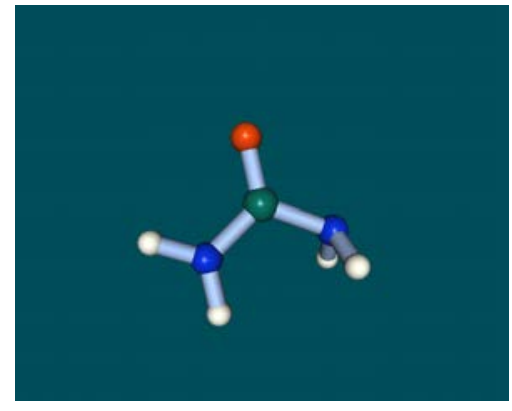

Figure 4.6: I - rank-2 saddle, $\mathrm{C}_{s}$ 
conformation E (Fig. 4.2), F (Fig. 4.3), and G (Fig. 4.4) are all rank-2 saddles. In these conformations, both nitrogen atoms take $s p^{3}$ hybridization. However, in conformation H (Fig. 4.5) and I (Fig. 4.6), only one nitrogen atom take $s p^{3}$ hybridization and another one is $s p^{2}$ hybridization (planar structure). This is clearly shown in Table 4.1 .

\begin{tabular}{|c|c|c|}
\hline & out-of-plane angle 1 & out-of-plane angle 2 \\
\hline Conformation E & 44.9 & 44.9 \\
\hline Conformation F & 44.9 & 45.5 \\
\hline Conformation G & 39.2 & 39.2 \\
\hline Conformation H & 43.8 & 0.0 \\
\hline Conformation I & 33.9 & 0.0 \\
\hline
\end{tabular}

Table 4.1: Out-of plane angle of different urea molecule conformations in its rotation PES

\subsection{Dipole moments}

An interesting observation is that the dipole moment of the molecule changes a lot during its internal rotation (Table 4.2). It is well-known that urea can dissolve in most solvents, including both polar and nonpolar solvents. The flexibility of urea molecule's geometry and the consequent change of its dipole moment could play important role in its excellent dissolving ability.

\begin{tabular}{|c|c|}
\hline & Dipole /debye \\
\hline Conformation A & 3.57 \\
\hline Conformation B & 4.26 \\
\hline Conformation E & 0.26 \\
\hline Conformation F & 2.81 \\
\hline Conformation G & 4.72 \\
\hline Conformation H & 2.42 \\
\hline Conformation I & 4.98 \\
\hline
\end{tabular}

Table 4.2: Dipole moments of important conformations in rotation PES of urea molecule 


\subsection{Internal rotation barrier}

\subsubsection{Early results}

The internal rotation barriers around the $\mathrm{C}-\mathrm{N}$ bonds of urea molecule has been measured by NMR method as $11.33 \mathrm{kcal} / \mathrm{mol}$ in dimethylformamide (DMF) solution [55], and $13 . \mathrm{k} \mathrm{kcal} / \mathrm{mol}$ in dimethylformamide-dimethyl sulfoxide solution [42]. In solid state, it was estimated as $25.9 \mathrm{kcal} / \mathrm{mol}$ [4] using infrared and Raman spectra data. However, no experimental data have been reported for urea molecule's gas phase rotational barrier. Early calculations by Kontoyianni and Bowen showed it as $16 \mathrm{kcal} / \mathrm{mol} 56$ ] using a rigid rotation model. Calculations including relaxation of the geometries lead to a barrier about $8 \mathrm{kcal} / \mathrm{mol}[28,40,41,57]$.

\subsubsection{Internal rotation barrier}

Both conformation $\mathrm{H}$ and I act as saddles in the internal rotation paths. Conformation $\mathrm{H}$ is $5.4 \mathrm{kcal} / \mathrm{mol}$ lower than conformation I in the PES (Table 4.3). So there exist two internal rotation barriers: higher one through conformation I is $13.5 \mathrm{kcal} / \mathrm{mol}$, and lower one through conformation $\mathrm{H}$ is $8.1 \mathrm{kcal} / \mathrm{mol}$.

However, it can be easily seen in PES plot (Fig. 4.1) that there always exist paths to avoid passing conformation I to connect energy minimum conformations (conformation $\mathrm{A}$ and $\mathrm{B})$.

\begin{tabular}{|c|c|c|c|}
\hline & Symmetry & Saddle Index & Energy $/ \mathrm{kcal} / \mathrm{mol}$ \\
\hline Conformation E & $C_{2 v}$ & rank-2 saddle & 29.8 \\
\hline Conformation F & $C_{s}$ & rank-2 saddle & 28.3 \\
\hline Conformation G & $C_{2 v}$ & rank-2 saddle & 31.6 \\
\hline Conformation H & $C_{s}$ & rank-1 saddle & 8.1 \\
\hline Conformation I & $C_{s}$ & rank-2 saddle & 13.5 \\
\hline
\end{tabular}

Table 4.3: Important conformations in rotation PES of urea molecule 


\subsubsection{Conjugation}

Due to the conjugation between CO double bond's $\pi$ electron pair and lone electron pairs of nitrogen atoms, the internal rotation barrier of urea molecule is higher than a typical free internal rotation barrier (the internal rotation barrier in ethane is about $2.9 \mathrm{kcal} / \mathrm{mol})$.

Rotation around the $\mathrm{C}-\mathrm{N}$ bond breaks the conjugation and significantly destabilizes the structure. This leads to substantial elongation of C-N bond (Table 4.4).

\begin{tabular}{|c|c|}
\hline & CN bond lengths $(\AA)$ \\
\hline Conformation A & $1.394,1.394$ \\
\hline Conformation E & $1.451,1.451$ \\
\hline Conformation F & $1.465,1.443$ \\
\hline Conformation G & $1.457,1.457$ \\
\hline Conformation H & $1.364,1.456$ \\
\hline Conformation I & $1.377,1.450$ \\
\hline
\end{tabular}

Table 4.4: CN bond lengths of important conformations in rotation PES of urea molecule

\subsubsection{Rotation barrier of floppy molecules}

One interesting thing about the rotation paths of urea molecule is that although conformation $\mathrm{H}$ is a rank-1 saddle, conformation I is actually a rank-2 saddle. There exist two decreasing normal modes for conformation I. One is along the rotational direction, another one is along the inversional direction of another side's amino group. Depending on which inversional direction it follows, any rotation path through conformation I will face a choice of two paths. One will lead to a global minimum (conformation A), and another one to a local minimum (conformation B).

And this observation should be generalized for all floppy molecules. Special attentions should be paid to exam whether or not there exist any high rank saddle points along the rotational path when calculating a floppy molecule's rotational energy barrier. if there exists any high rank saddle point along the rotational path, there will be 
more than one rotational paths the molecule can choose.

\subsection{Fitting rotation PES}

Rotation energy barrier is higher than inversion barrier, therefore while urea molecule undergoes rotation motion, it could undergo inversion motion at the same time. The inversion variables have to be included also while fitting rotation PES.

Rotation PES was fitted using rotation variables $\theta_{1}$ and $\theta_{2}$ together with two inversion variables $\varphi_{1}$ and $\varphi_{2}$ (see section 3.1):

$$
\begin{array}{cc}
\theta_{1}=\frac{\tau_{5}+\tau_{6}}{2}, & \theta_{2}=\frac{\tau_{7}+\tau_{8}}{2} \\
\varphi_{1}=\left(\tau_{5}-\tau_{6}\right)-\pi, & \varphi_{2}=\left(\tau_{7}-\tau_{8}\right)-\pi
\end{array}
$$

It was fitted into a polynomial up to order 6 :

$$
V\left(\varphi_{1}, \varphi_{2}, \theta_{1}, \theta_{2}\right)=\sum_{a+b+c+d \leq 6} f_{a b c d}\left(\varphi_{1}\right)^{a}\left(\varphi_{2}\right)^{b}\left(\cos \theta_{1}\right)^{c}\left(\cos \theta_{2}\right)^{d}
$$

All data from both inversion PES (1800 data) and rotation PES ( 2088) were used. Totally 210 terms were generated, but only 110 coefficients are independent due to symmetry condition:

$$
f_{a b c d}=f_{b a d c}
$$

All independent coefficients are listed in Table B.1 in Appendix B. 


\section{Chapter 5}

\section{Symmetry Analysis}

\subsection{Complete Permutation-inversion Group}

Any rearrangement of identical nuclei in a urea molecule leads to a new but energetically equivalent conformation. The total number of these conformations equals to the order of the full permutation-inversion group of urea molecule, which is a direct product of the permutation groups and the inversion group $\epsilon$ :

$$
G=S_{N} \otimes S_{H} \otimes \epsilon=2 ! \times 4 ! \times 2=96
$$

where $S_{N}$ and $S_{H}$ are the groups of permutations of nitrogen atoms and hydrogen atoms.

\subsection{Subsurfaces of PES}

However, not all these conformations are accessible to each other in the PES due to the existing high barriers (which corresponding to bond breaking). For example, of 48 energetically equivalent global energy minimum conformations, only 8 of them are accessible to each other (Fig 5.1). 


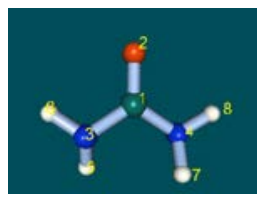

(a) $\mathrm{A}_{1}$

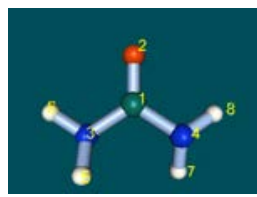

(e) $\mathrm{A}_{1}^{\prime}$

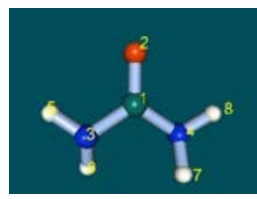

(b) $\mathrm{A}_{2}$

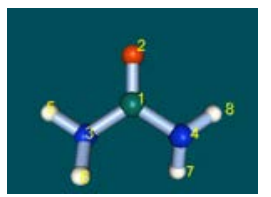

(f) $\mathrm{A}_{2}^{\prime}$

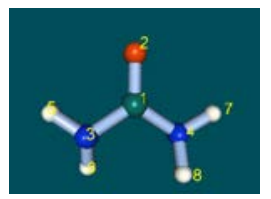

(c) $\mathrm{A}_{3}$

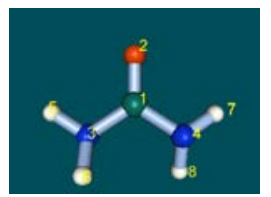

(g) $\mathrm{A}_{3}^{\prime}$

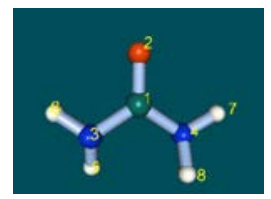

(d) $\mathrm{A}_{4}$

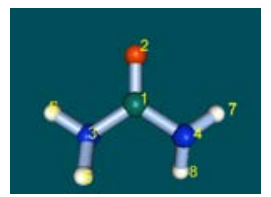

(h) $\mathrm{A}_{4}^{\prime}$

Figure 5.1: Eight energetically equivalent global energy minimum conformations of urea molecule which are accessible to each others.

The whole PES will split into several subsurfaces which are seperated by insurmountable energy barriers. The number of subsurfaces can be find from the order of complete permutation-inversion group and Longuet-Higgins group:

$$
n=\frac{\text { order of complete permutation-inversion group }}{\text { order of Longuet-Higgins group }}
$$

\subsection{Longuet-Higgins Group}

For non-rigid molecules, Longuet-Higgins showed that the most useful groups should consist all "feasible" permutations of the various identical nuclei in the molecule, with or without an accompanying inversion of the positions of all particles in the centre of mass [58. In order to be "feasible", a permutation-inversion operation should be carried out without passing over a insurmountable energy barrier. By considering LonguetHiggins group instead of point group of the molecule, more valuable information, such as selection rules, can be provided.

For a rigid molecule, its Longuet-Higgins group is the same as its point group. But for a non-rigid molecule, its point group is a subgroup of its Longuet-Higgins group. 


\subsection{Longuet-Higgins Group of Urea}

All feasible permutation-inversion operations within urea molecule can are listed below (atom labelled as Fig. 5.2):

$$
\begin{gathered}
E,(34)(5768),(34)(5867),(56)(78),(78),(56),(34)(57)(68),(34)(58)(67) \\
E^{*},(34)(5768)^{*},(34)(5867)^{*},(56)(78)^{*},(78)^{*},(56)^{*},(34)(57)(68)^{*},(34)(58)(67)^{*}
\end{gathered}
$$

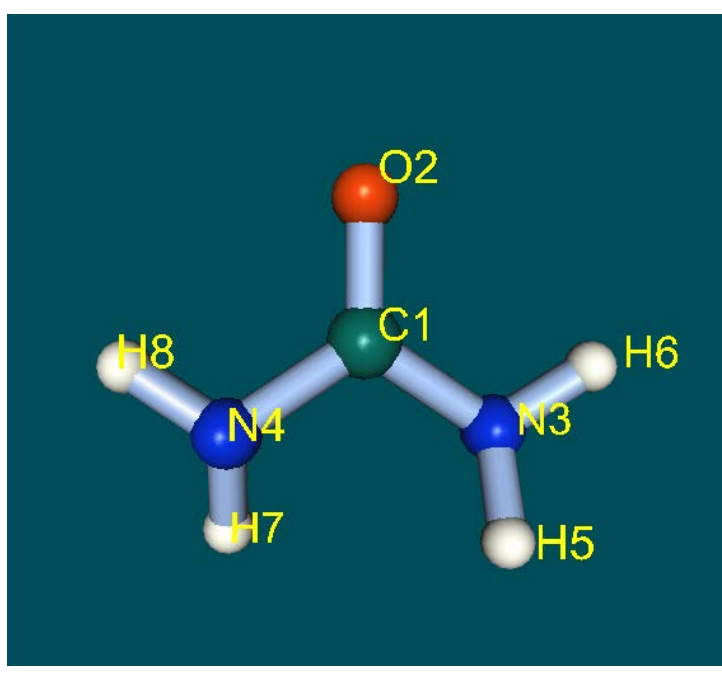

Figure 5.2: Atom numbering for urea molecule's permutation-inversion operation - Plotted using Facio software

This is a group of order 16. Many operations which are not present in its point group are allowed due to inversion and rotation motions within urea molecule. For example, permutation (78) and $(78)^{*}$ are only feasible when rotation around $\mathrm{C}_{1} \mathrm{~N}_{4}$ bond and inversion of $\mathrm{N}_{4} \mathrm{H}_{7} \mathrm{H}_{8}$ amino group are considered.

Therefore, the whole PES of urea will split into 6 subsurfaces:

$$
6=\frac{96}{16}
$$

The subsurfaces were characterized by an order-16 Longuet-Higgins group, which 
is a group isomorphous to group $D_{4 h}$. All 16 permutation-inversion operators of the group are described in Fig. 5.3 and Fig. 5.4. The character table of the group is given in Table 5.1. The symbols + and - have been used to indicate the parity (the character with respect to the inversion $\left.E^{*}\right)$ of each representation.

\subsection{Tunneling}

After obtaining urea's Longuet-Higgins group, many valuable information can be provided. One of the most important applications is its energy level degeneration due to tunneling.

The global energy minimum conformation of urea molecule has a symmetry of $C_{2}$ (Fig. 3.2 which corresponds to the permutation $(34)(57)(68)$ or $(34)(58)(67)$. If the internal inversion and internal rotation are impossible for urea molecules, there will exist eight distinct isoenergetic conformations. They are non-superimposable because of permutation of identical nuclei and inversion about center of mass. Each of these eight conformations has its own vibration-rotation wavefunction and they are either symmetric $\left(A_{1}\right)$ or antisymmetric $\left(A_{2}\right)$ about the $C_{2}$ axis. These eight wavefunctions will generate reducible representations of the Longuet-Higgins group. The characters of the reducible representation can be determined.

Each operator of the group, except $E,(34)(57)(68)$ and (34)(58)(67), transforms one conformation into another distinct conformation. This can be seen by applying these operators on each of eight conformations in Fig. 5.3. None of them will reproduce themself. The characters of these operator are all zero.

Permutation $(34)(57)(68)$ and $(34)(58)(67)$ each induces a $C_{2}$ rotation for four of the eight conformations or wavefunctions. The sign of characters are determined by these wave functions' symmetry with respect to the $C_{2}$ axis.

The characters of reducible representations therefore can be derived from above 


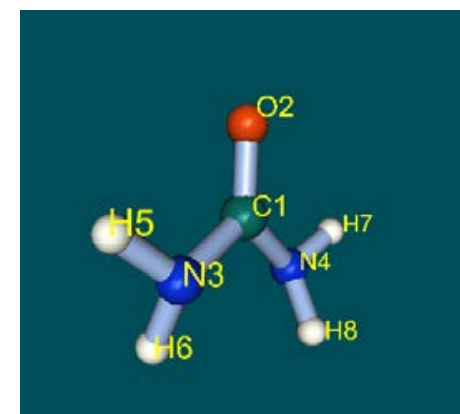

1: $\mathrm{E}$

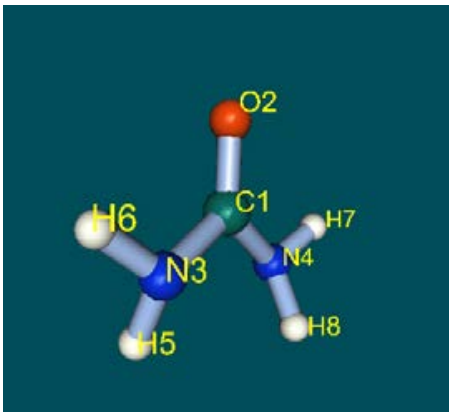

3: $(56)$

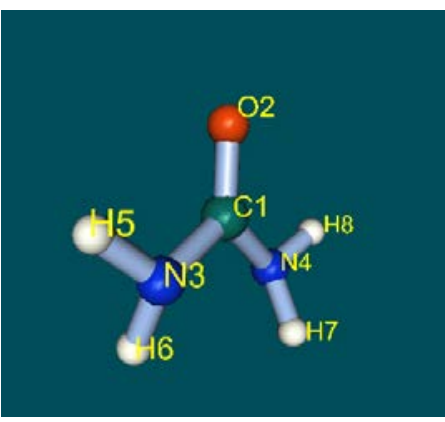

5: (78)

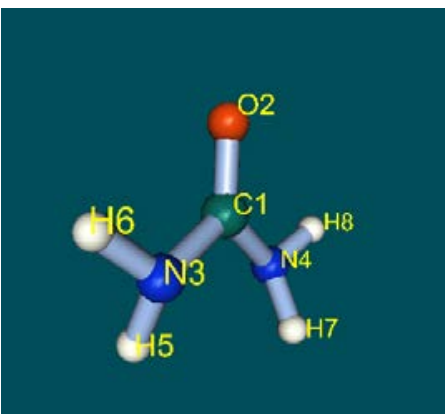

$7:(56)(78)$

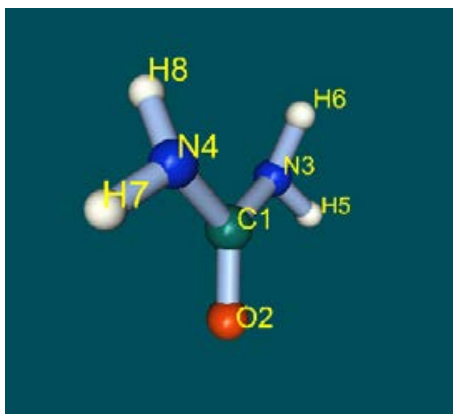

2: $\mathrm{E}^{*}$

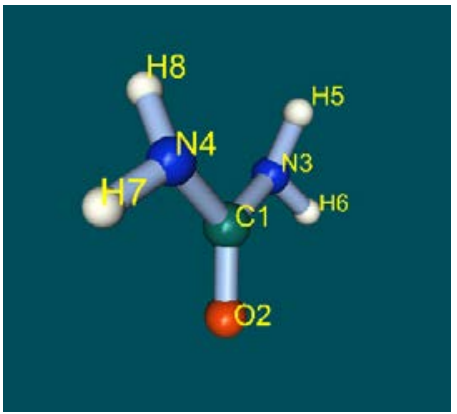

4: $(56)^{*}$

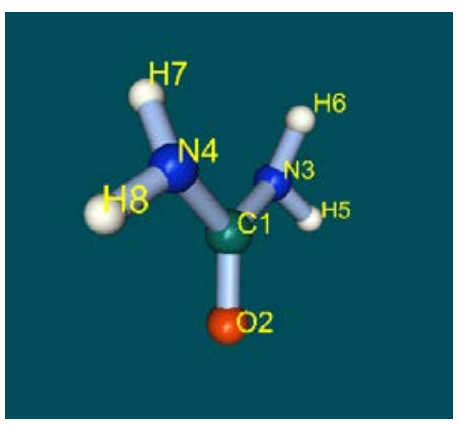

6: $(78)^{*}$

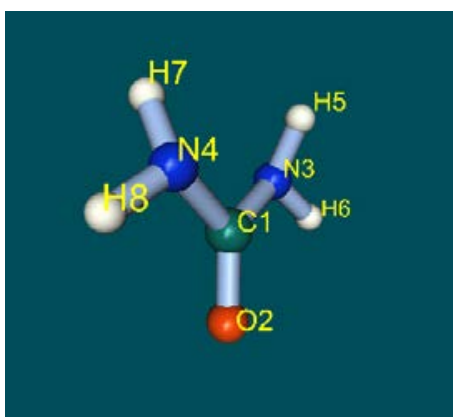

8: $(56)(78)^{*}$

Figure 5.3: Elements of urea's permutation group: cont. 


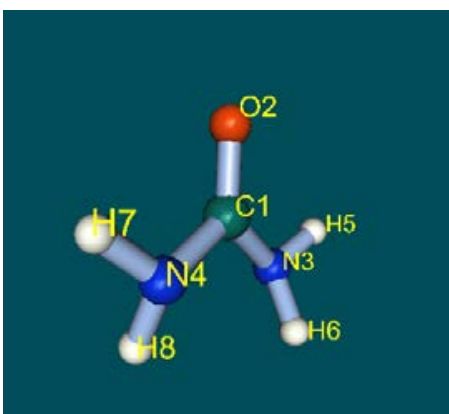

9: $(34)(57)(68)$

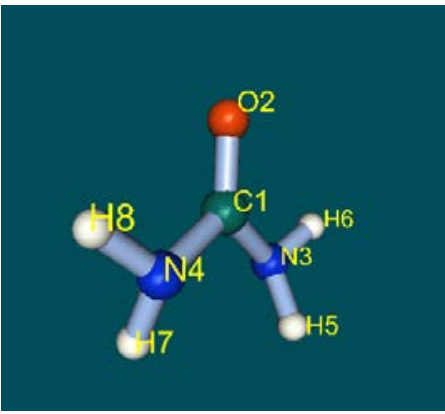

11: $(34)(58)(67)$

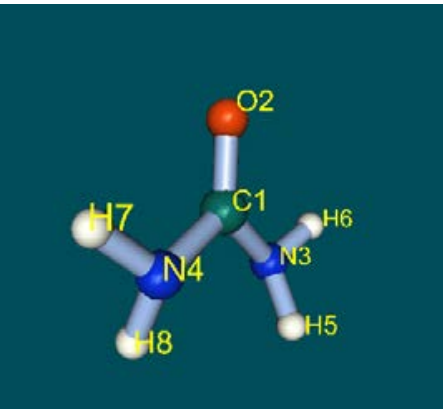

13: $(34)(5768)$

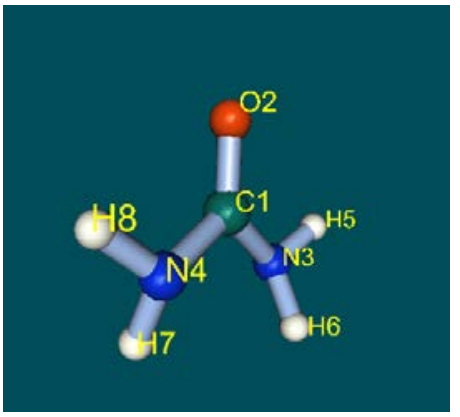

15: $(34)(5867)$

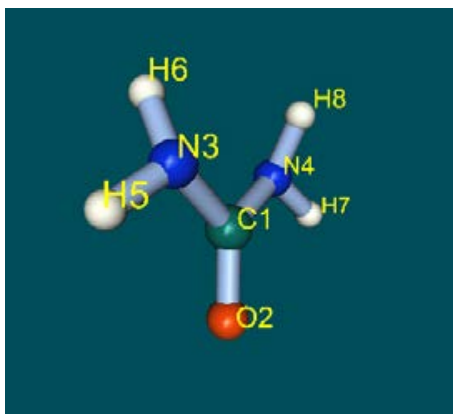

10: $(34)(57)(68)^{*}$

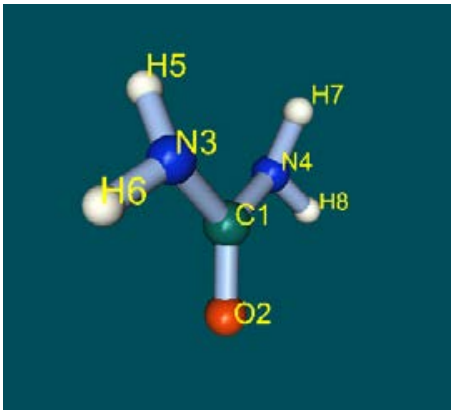

12: $(34)(58)(67)^{*}$

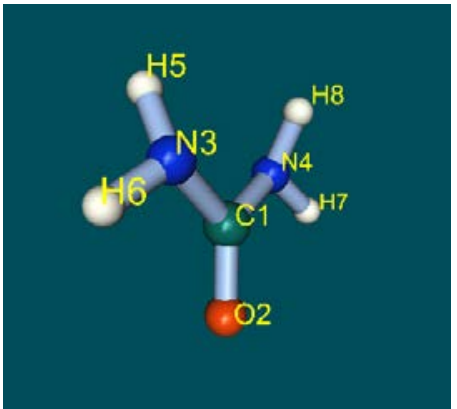

14: $(34)(5768)^{*}$

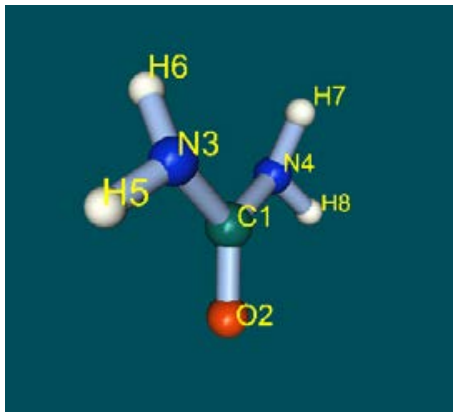

16: $(34)(5867)^{*}$

Figure 5.4: Elements of urea's permutation group: contd. 


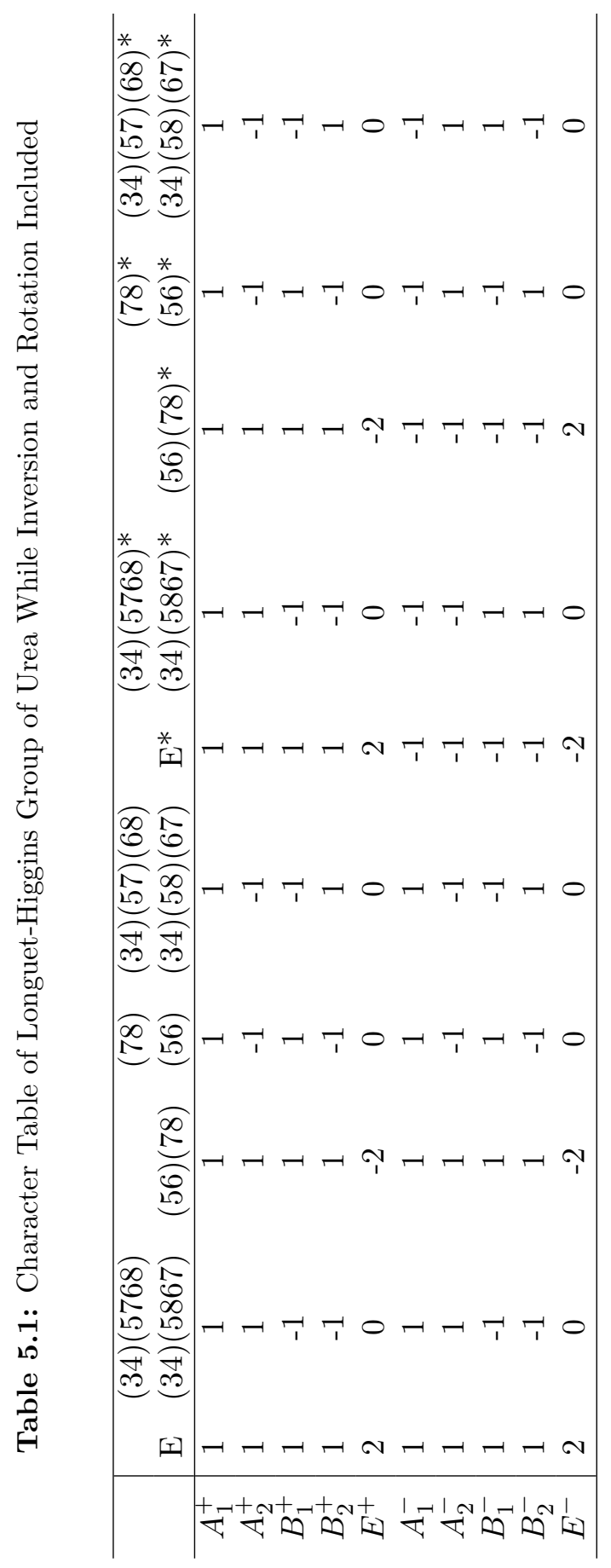


discussions. They are listed in Table 5.2 .

Table 5.2: Reducible representation of symmetric and antisymmetric levels

\begin{tabular}{|c|c|c|c|c|c|c|c|c|c|c|}
\hline & Symmetry under $C_{2}$ & & & & Character & & & & & \\
\hline & & & & & $(34)(57)(68)$ & & & & & \\
\hline & $E$ & & & & $(34)(58)(67)$ & & & & & \\
\hline$A_{1}$ & 8 & 0 & 0 & 0 & 4 & 0 & 0 & 0 & 0 & 0 \\
\hline$A_{2}$ & 8 & 0 & 0 & 0 & -4 & 0 & 0 & 0 & 0 & 0 \\
\hline
\end{tabular}

Since the energy barriers of internal inversion and internal rotation are reachable for urea molecule, each energy level of it will be split into several sublevels due to the tunneling between the isoenergetic conformations. A symmetric level $\left(A_{1}\right)$ will split into 6 sublevels:

$$
A_{1}^{+}+B_{2}^{+}+E^{+}+A_{1}^{-}+B_{2}^{-}+E^{-}
$$

and an antisymmetric one $\left(A_{2}\right)$ into:

$$
A_{2}^{+}+B_{1}^{+}+E^{+}+A_{2}^{-}+B_{1}^{-}+E^{-}
$$

As shown above, including inversion and rotation will change the way a level being assigned. The levels belonging to the same species under the point group of the molecule will belong to different species due to internal rotation and inversion.

\subsection{Electric Dipole Selection Rules}

Electric dipole selection rules of urea can also be obtained by analyzing its LonguetHiggins group.

The symmetry of the electric dipole is like $A_{1}^{-}$: it is invariant to all permutations without an inversion about the center of mass but changes sign to all permutations 
with an inversion. Therefore, the selection rules of the electric dipole is:

$$
+\leftrightarrow-\quad \Gamma_{i} \leftrightarrow \Gamma_{i}
$$

That is, $A_{1}^{+} \leftrightarrow A_{1}^{-}, B_{1}^{+} \leftrightarrow B_{1}^{-}, A_{2}^{+} \leftrightarrow A_{2}^{-}, B_{2}^{+} \leftrightarrow B_{2}^{-}$, and $E^{+} \leftrightarrow E^{-}$.

\subsection{Symmetry of inversion PES}

If only two inversion motions were considered, the two inversion motions around nitrogen atoms will make the inversion about the molecule's center of mass feasible. There will be tatally four feasible permutation-inversion operations:

$$
E,(34)(57)(68), E^{*},(34)(57)(68)^{*},
$$

and it is a group isomorphous with $C_{2 h}$ group. Its character table is given in Table 5.3 .

Table 5.3: Character Table of Longuet-Higgins Group of Urea While Only Inversion Included

\begin{tabular}{c|cccc}
\hline & $\mathrm{E}$ & $(34)(57)(68)$ & $\mathrm{E}^{*}$ & $(34)(57)(68)^{*}$ \\
\hline$A^{+}$ & 1 & 1 & 1 & 1 \\
$A^{-}$ & 1 & 1 & -1 & -1 \\
$B^{+}$ & 1 & -1 & 1 & -1 \\
$B^{-}$ & 1 & -1 & -1 & 1 \\
\hline
\end{tabular}

\subsection{Symmetry of rotation PES}

If only two rotation motions were considered, there will be totally four feasible permutationinversion operations:

$$
E,(56)(78),(34)(57)(68),(34)(58)(67)
$$


and it is a group isomorphous with $C_{2 h}$ group. Its character table is given in Table 5.4.

Table 5.4: Character Table of Longuet-Higgins Group of Urea While Only Rotation Included

\begin{tabular}{c|cccc}
\hline & $\mathrm{E}$ & $(34)(57)(68)$ & $(56)(78)$ & $(34)(58)(67)$ \\
\hline$A^{+}$ & 1 & 1 & 1 & 1 \\
$A^{-}$ & 1 & 1 & -1 & -1 \\
$B^{+}$ & 1 & -1 & 1 & -1 \\
$B^{-}$ & 1 & -1 & -1 & 1 \\
\hline
\end{tabular}

While both inversion and rotation motions were included, we will have a group which is direct product of $C_{2}$ and $C_{2}$, a group of order 16 , which is confirmed in section 5.4 .

\subsection{Graphical Description}

A graphical description method was proposed to represent floppy molecules' PES by Simons et al. [59]. In the graphical description, vertices denote energy minima and edges connecting the vertices describe energetically accessible paths connecting such minima. The graphical description is useful to understand the topology of high dimensional PES. However, cases in which two or more accessible paths can interconnect equivalent minima or in which nonequivalent energy minima exist were not developed. Urea's PES, in which there exist global and local minima, and multi-paths connecting these minima, could serve as a perfect example to extend the application.

\subsection{Graphical Description of Urea PES}

As analyzed in section 5.4, urea's PES splits into 6 subsurfaces, each of them can be characterized by its Longuet-Higgins group, a group of order 16. The number of each important conformations in one subsurface can be calculated and listed in Table 5.5 : 


$$
n=\frac{\text { order of Longuet-Higgins group }}{\text { order of conformation's symmetry }}
$$

\begin{tabular}{|c|c|c|}
\hline & Symmetry & Number in one subsurface \\
\hline Conformation A & $C_{2}$ & 8 \\
\hline Conformation B & $C_{s}$ & 8 \\
\hline Conformation C & $C_{2 v}$ & 4 \\
\hline Conformation D & $C_{1}$ & 16 \\
\hline Conformation E & $C_{2 v}$ & 4 \\
\hline Conformation F & $C_{s}$ & 8 \\
\hline Conformation G & $C_{2 v}$ & 4 \\
\hline Conformation H & $C_{s}$ & 8 \\
\hline Conformation I & $C_{s}$ & 8 \\
\hline
\end{tabular}

Table 5.5: Number of important conformations in PES of urea molecule

To understand the topology of PES, the relationship between the eight accessible conformation A in Fig 5.1 can be represented as a cube (Fig,5.5).

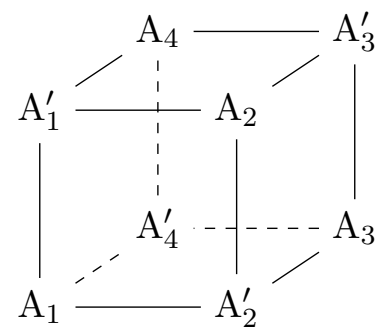

Figure 5.5: Graphic description of connections on PES of urea molecule

The top four conformations are connected to bottom four via internal inversion. As Fig 3.1 shown, conformations B, C, and D lie in these pathways. There are 2 conformation B, 1 conformation $\mathrm{C}$, and 4 conformation $\mathrm{D}$ in each of these inversion connections (Fig 5.6 ), and we have totally four these inversion connections (Fig 5.5 ). Therefore, all 8 conformation B, 4 conformation C, and 16 conformation D from Table 5.5 are located.

It should be pointed out that for local minima conformation $\mathrm{B}$, there exists a similar 


$$
\mathrm{A} \underset{\mathrm{D}_{3}-\mathrm{B}_{2}-\mathrm{D}_{4}-\mathrm{B}_{1}-\mathrm{D}_{2}}{\succ} \mathrm{A}^{\prime}
$$

Figure 5.6: Inversion paths on PES of urea molecule

cubic graphical structure just like global minima A in Fig. 5.5. The two cubic structures interweave together, and their connections were partially represented in Fig. 5.6.

If only one amino group makes the internal rotation, the path will go through conformation $\mathrm{H}$ or I, depending on the direction of rotation (Fig 5.7 respectively.

$$
\begin{aligned}
& \mathrm{A}_{1}^{\prime}=\mathrm{H}_{\mathrm{I}^{\prime}}=\mathrm{A}_{2} \\
& \mathrm{~A}_{1}=\mathrm{H}=\mathrm{A}_{2}^{\prime}
\end{aligned}
$$

Figure 5.7: One-side rotation paths on PES of urea molecule

There are 1 conformation $\mathrm{H}$ and 1 conformation I in each of these one-side rotation connections, and we have totally eight these one-side paths (Fig 5.5). Therefore, all 8 accessible conformation $\mathrm{H}$ and 8 accessible conformation I from Table 5.5 are located.

If both amino groups make the internal rotation, the path will go through conformation $\mathrm{E}, \mathrm{F}$, or $\mathrm{G}$, depending on different combinations of the direction of two rotations (Fig 5.8).

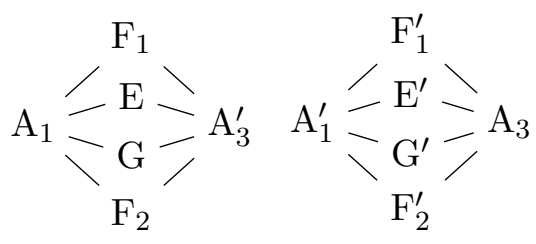

Figure 5.8: Two-side rotation paths on PES of urea molecule

There are 1 conformation E, 2 conformation F, and 1 conformation G in each of these two-side rotation connections. Since we have totally four these two-side paths 
(four diagonals in Fig,5.5), all 4 accessible conformation E, 8 accessible conformation $\mathrm{F}$, and 4 accessible conformation $\mathrm{G}$ from Table 5.5 are located. 


\section{Chapter 6}

\section{Conclusion}

In this study, the four-dimensional potential energy surface (PES) for the double internal inversion and rotation in urea was determined using fully optimized MP2/aug-ccpVDZ calculations using new introduced inversion and rotation variables.

Calculations of totally 3888 conformations selected by symmetry considerations and energy criteria were performed. They were then fitted to a polynomial up to order six. The total number of the independent coefficients are 110 .

The most significant properties of this potential, including the inversion barrier and the rotation barrier, were analyzed. The topology of the PES was characterized using symmetry analysis. A graphical description method, suggested by Simons et al. [59], was extended to describe the four-dimensional PES. 
Appendices 


\section{Appendix A}

\section{Potential energy of urea fitting}

\section{around conformation D}

Totally 1800 conformations were chosen randomly in the range of $\tau_{1}= \pm 5, \tau_{2}^{\prime}= \pm 5, \tau_{3}=$ $\pm 5, \tau_{4}^{\prime}= \pm 5$ and fitted into the following form:

$$
V=\sum_{a+b+c+d \leq 4} f_{a b c d}\left(\tau_{1}\right)^{a}\left(\tau_{2}^{\prime}\right)^{b}\left(\tau_{3}\right)^{c}\left(\tau_{4}^{\prime}\right)^{d}
$$

Totally 75 terms were generated and coefficients were listed in Table A.1 (units in $\left.\mathrm{cm}^{-1}\right)$. Root mean square error is 0.007619558 , and coefficient of determination $\left(\mathrm{R}^{2}\right)$ is 0.999999715 . 


\begin{tabular}{|r|r|r|r|r|r|}
\hline$f_{0000}$ & 0.037 & $f_{1000}$ & 0.003 & $f_{0100}$ & 0.008 \\
$f_{0010}$ & 0.003 & $f_{0001}$ & 0.008 & $f_{2000}$ & 1483.687 \\
$f_{0200}$ & 129.158 & $f_{0020}$ & 1483.687 & $f_{0002}$ & 129.158 \\
$f_{1100}$ & 3248.440 & $f_{1010}$ & -1664.804 & $f_{1001}$ & -704.814 \\
$f_{0110}$ & -704.814 & $f_{0101}$ & -1400.090 & $f_{0011}$ & 3248.440 \\
$f_{3000}$ & -0.839 & $f_{0300}$ & -0.333 & $f_{0030}$ & -0.839 \\
$f_{0003}$ & -0.333 & $f_{2100}$ & -1.750 & $f_{2010}$ & 0.083 \\
$f_{2001}$ & -0.914 & $f_{1200}$ & -1.292 & $f_{1020}$ & 0.083 \\
$f_{1002}$ & 0.948 & $f_{0210}$ & 0.948 & $f_{0201}$ & -1.082 \\
$f_{0120}$ & -0.914 & $f_{0102}$ & -1.082 & $f_{0021}$ & -1.750 \\
$f_{0012}$ & -1.292 & $f_{1110}$ & -1.052 & $f_{1101}$ & 2.304 \\
$f_{1011}$ & -1.052 & $f_{0111}$ & 2.304 & $f_{4000}$ & -540.930 \\
$f_{0400}$ & -99.630 & $f_{0040}$ & -540.930 & $f_{0004}$ & -99.630 \\
$f_{3100}$ & -1684.233 & $f_{3010}$ & 613.215 & $f_{3001}$ & 388.047 \\
$f_{1300}$ & -1329.777 & $f_{1030}$ & 613.215 & $f_{1003}$ & 260.214 \\
$f_{0310}$ & 260.214 & $f_{0301}$ & 275.507 & $f_{0130}$ & 388.047 \\
$f_{0103}$ & 275.507 & $f_{0031}$ & -1684.233 & $f_{0013}$ & -1329.777 \\
$f_{2200}$ & 2326.416 & $f_{2110}$ & 39.098 & $f_{2101}$ & -6.709 \\
$f_{2020}$ & 1020.014 & $f_{2011}$ & -14.738 & $f_{2002}$ & 484.316 \\
$f_{1210}$ & 262.711 & $f_{1201}$ & 540.704 & $f_{1120}$ & -14.738 \\
$f_{1102}$ & 284.688 & $f_{1021}$ & 39.098 & $f_{1012}$ & 262.711 \\
$f_{0220}$ & 484.316 & $f_{0211}$ & 284.688 & $f_{0202}$ & 653.430 \\
$f_{0121}$ & -6.709 & $f_{0112}$ & 540.704 & $f_{0022}$ & 2326.416 \\
$f_{1111}$ & 591.299 & & & & \\
\hline
\end{tabular}

Table A.1: Coefficients of fitted PES of urea around conformation D - units in $\mathrm{cm}^{-1}$ 


\section{Appendix B}

\section{Coefficients of fitted rotation}

\section{PES of urea}

Totally 3888 data were used for the fitting:

$$
V\left(\varphi_{1}, \varphi_{2}, \theta_{1}, \theta_{2}\right)=\sum_{a+b+c+d \leq 6} f_{a b c d}\left(\varphi_{1}\right)^{a}\left(\varphi_{2}\right)^{b}\left(\cos \theta_{1}\right)^{c}\left(\cos \theta_{2}\right)^{d}
$$

210 terms were generated but only 110 coefficients are independent due to symmetry restriction:

$$
f_{a b c d}=f_{b a d c}
$$

They are listed in Table B.1 (units in $\mathrm{cm}^{-1}$ ). Root mean square error is 85.0, and coefficient of determination $\left(\mathrm{R}^{2}\right)$ is 0.999095056 . 


\begin{tabular}{|c|c|c|c|c|c|}
\hline$f_{0000}$ & -589284.4745 & $f_{0001}$ & -316105.9067 & $f_{0002}$ & .2612 \\
\hline$f_{0003}$ & -665583.5241 & $f_{0004}$ & -338222.2309 & $f_{0005}$ & 112308.6759 \\
\hline$f_{0006}$ & -66628.54711 & $f_{0011}$ & 188727.4949 & $f_{0012}$ & 160638.2717 \\
\hline$f_{0022}$ & -212421.8051 & $f_{0031}$ & 102531.1903 & $f_{0032}$ & -597173.7565 \\
\hline$f_{0033}$ & -268192.9984 & $f_{0041}$ & 489049.3211 & $f_{0042}$ & 37633.10974 \\
\hline$f_{0051}$ & 201174.101 & $f_{0100}$ & 1824880.441 & $f_{0110}$ & 616120.382 \\
\hline$f_{0113}$ & 39366.28683 & $f_{0120}$ & 223903.6325 & $f_{0121}$ & -904573.4736 \\
\hline$f_{0122}$ & 112712.7104 & $f_{0123}$ & 347527.1727 & $f_{0130}$ & 444061.1481 \\
\hline$f_{0140}$ & 468114.0812 & $f_{0211}$ & 309750.9547 & $f_{0212}$ & -973713.1468 \\
\hline$f_{0300}$ & 581971.3373 & $f_{0301}$ & -1784499.326 & $f_{0302}$ & -977449.3539 \\
\hline$f_{0303}$ & 166617.8839 & $f_{0310}$ & -119082.4591 & $f_{0320}$ & 179421.8147 \\
\hline$f_{0330}$ & -183524.4124 & $f_{1005}$ & -257696.0058 & $f_{1010}$ & 928130.4483 \\
\hline$f_{1011}$ & -384347.5212 & $f_{1013}$ & -125534.9922 & $f_{1014}$ & -231231.1794 \\
\hline$f_{1020}$ & 352281.7771 & $f_{1021}$ & 352818.8445 & $f_{1023}$ & 394729.2363 \\
\hline$f_{1030}$ & 1569 & $f_{1040}$ & 1932 & $f_{1041}$ & 3.6024 \\
\hline$f_{1050}$ & -27981.60488 & $f_{1100}$ & -6275863.145 & $f_{1101}$ & -2504044.626 \\
\hline$f_{1102}$ & -783534.6503 & $f_{1103}$ & -1161281.868 & $f_{1104}$ & -142183.5924 \\
\hline$f_{1111}$ & 528528.44 & $f_{1112}$ & 42337.35737 & $f_{1122}$ & -286271.2633 \\
\hline$f_{1131}$ & 77428 & $f_{1200}$ & 5814 & $f_{1210}$ & 316.27 \\
\hline$f_{1220}$ & 5588 & $f_{1221}$ & -47422 & $f_{1230}$ & .5888 \\
\hline$f_{1311}$ & -98633 & $f_{1400}$ & 7218 & $f_{1401}$ & -999006.8037 \\
\hline$f_{1410}$ & -136597.5601 & $f_{1500}$ & -600387.1065 & $f_{2000}$ & -1492058.38 \\
\hline$f_{2001}$ & -370345.5221 & $f_{200}$ & -74740.82431 & $f_{2003}$ & 386215.2914 \\
\hline$f_{2004}$ & -3165 & $f_{2010}$ & 15131 & $f_{2012}$ & 1473069.337 \\
\hline$f_{2013}$ & 22937 & $f_{2020}$ & 11034 & $f_{2022}$ & 98397.82223 \\
\hline$f_{2030}$ & -1177384.428 & $f_{2031}$ & -393308.4163 & $f_{2040}$ & 199052.3747 \\
\hline$f_{2110}$ & 2036068.863 & $f_{2111}$ & -161648.3444 & $f_{2120}$ & -921938.6401 \\
\hline$f_{2121}$ & 453305.3069 & $f_{2130}$ & 1157237.303 & $f_{2200}$ & -5816963.753 \\
\hline$f_{2201}$ & -2693913.713 & $f_{2202}$ & 12171: & $f_{2211}$ & -4017.750715 \\
\hline$f_{2300}$ & 1078512.252 & $f_{2301}$ & 1249051.375 & $f_{2310}$ & 128026.7765 \\
\hline$f_{3011}$ & -259386.6387 & $f_{3012}$ & -585150.5986 & $f_{3021}$ & 415919.0078 \\
\hline$f_{3100}$ & -1187210.378 & $f_{31}$ & 65643.48953 & $f_{3102}$ & 9281.532149 \\
\hline$f_{3110}$ & 572421.3938 & $f_{3120}$ & 690656.3945 & $f_{3300}$ & -436432.4202 \\
\hline$f_{4000}$ & -1307788.729 & $f_{4001}$ & 235137.2199 & $f_{4002}$ & -159964.6918 \\
\hline$f_{4010}$ & 1231953.416 & $f_{4011}$ & 258422.6603 & $f_{4020}$ & -84556.03438 \\
\hline$f_{4200}$ & 219207.2752 & $f_{5000}$ & 980752.1124 & $f_{5001}$ & -109955.6208 \\
\hline 5010 & -145115.4992 & $f_{6000}$ & -68479.1996 & & \\
\hline
\end{tabular}

Table B.1: Coefficients of fitted rotation PES of urea - units in $\mathrm{cm}^{-1}$ 


\section{References}

[1] T. O. Lipman, "Wohler's preparation of urea and the fate of vitalism," Journal of Chemical Education, vol. 41, pp. 452-458, 1964. 1]

[2] T. T. Herskovits, H. Jaillet, and B. Gadegbeku, "On the structural stability and solvent denaturation of proteins. ii. denaturation by the ureas," Journal of Biological Chemistry, vol. 245 , pp. 4544-4550, 1970. 1

[3] T. T. Herskovits, H. Jaillet, and A. T. DeSena, "On the structural stability and solvent denaturation of proteins. iii. denaturation by the amides," Journal of Biological Chemistry, vol. 245 , pp. $6511-6517,1970.1$

[4] H. S. Frank and F. Franks, "Structural approach to the solvent power of water for hydrocarbons; urea as a structure breaker," Journal of Chemical Physics, vol. 48, pp. 4746-4757, 1968. 1., 2

[5] J. Tirado-Rives, M. Orozco, and W. L. Jorgensen, "Molecular dynamics simulations of the unfolding of barnase in water and $8 \mathrm{~m}$ aqueous urea," Biochemistry, vol. 36, p. 73137329, 1997. 1

[6] K. A. Sharp, B. Madan, E. Manas, and J. M. Vanderkooi, "Water structure changes induced by hydrophobic and polar solutes revealed by simulations and infrared spectroscopy," Journal of Chemical Physics, vol. 114, pp. 1791-1796, 2001. 1. 2

[7] G. Jakli and W. A. V. Hook, "Excess thermodynamic properties of h2o and d2o solutions of tetramethylurea, an azeotropic system. vapor pressures, excess vapor pressures, and vapor pressure isotope effects," Journal of Chemical and Engineering Data, vol. 46, p. 777781, 2001. 1, 2 
[8] D. Tobi, R. Elber, and D. Thirumalai, "The dominant interaction between peptide and urea is electrostatic in nature a molecular dynamics simulation study," Biopolymers, vol. 68, pp. 359-369, 2003. 1

[9] A. K. Soper, E. W. C. Jr., and A. Luzar, "Impact of urea on water structure: a clue to its properties as a denaturant?," Biophysical Chemistry, vol. 105, pp. 649-666, 2003. 1. 2

[10] Y. L. A. Rezus and H. J. Bakker, "Effect of urea on the structural dynamics of water," The Proceedings of the National Academy of Sciences, vol. 103, pp. 18417-18420, 2006. 1 . 2

[11] R. Zangi, R. Zhou, and B. J. Berne, "Ureas action on hydrophobic interactions," Journal of the American Chemical Society, vol. 131, p. 15351541, 2009. 1

[12] J. C. Ahluwalia, S. Subramanian, T. S. Sarma, and D. Balasubramanian, "Effects of the urea-guanidinium class of protein denaturants on water structure: heats of solution and proton chemical shift studies," Journal of Physical Chemistry, vol. 75, pp. 815-820, 1971. 2

[13] F. Franks and D. S. Reid, Water: A Comprehensive Treatise. Plenum: New York, 1973. 2

[14] G. E. Walrafen, "Raman spectral studies of the effects of urea and sucrose on water structure," Journal of Chemical Physics, vol. 44, pp. 3726-3727, 1966. 2

[15] D. V. Beauregard and R. E. Barrett, "Ultrasonics and water structure in urea solutions," Journal of Chemical Physics, vol. 49, pp. 5241-5244, 1968. 2

[16] R. Stokes, "Thermodynamics of aqueous urea solutions," Australian Journal of Chemistry, vol. 20, pp. 2087-2100, 1967. 2

[17] A. Idrissi, "Molecular structure and dynamics of liquids - aqueous urea solutions," Spectrochimica Acta Part A: Molecular and Biomolecular Spectroscopy, vol. 61, pp. 1-17, 2005. 2.3

[18] E. G. Finer, F. Franks, and M. J. Tait, "Nuclear magnetic resonance studies of aqueous urea solutions," Journal of the American Chemical Society, vol. 94, p. 44244429, 1972. 2, 3 
[19] H. Tanaka, H. Touhara, K. Nakanishi, and N. Watanabe, "Computer experiment on aqueous solution. iv. molecular dynamics calculation on the hydration of urea in an infinitely dilute aqueous solution with a new ureawater pair potential," Journal of Chemical Physics, vol. 80 , pp. $5170-5186,1984.2$

[20] W. D. Kumler and G. M. Fohlen, "The dipole moment and structure of urea and thiourea," J. Am. Chem. Soc., vol. 64, pp. 1944-1948, 1942. 3, 21

[21] A. Caron, "Redetermination of thermal motion and interatomic distances in urea," Acta Crystallographica, Section B: Structural Science, vol. 25, pp. 404-404, 1969. 3

[22] S. Swaminathan and B. M. Craven, "Theoretical and experimental studies of the charge density in urea," Acta Crystallographica Section B, vol. 40, pp. 398-404, 1984. 3

[23] J. E. Worsham, H. A. Levy, and S. W. Peterson, "The positions of hydrogen atoms in urea by neutron diffraction," Acta Crystallographica, vol. 10, pp. 319-323, 1957. 3

[24] A. W. Pryor and P. L. Sanger, "Collection and interpretation of neutron ditfraetion measurements on urea," Acta Crystallographica, Section A: Foundations of Crystallography, vol. 26, pp. 543-558, 1970. 3

[25] S. Swaminathan, B. M. Craven, and R. K. McMullan, "The crystal structure and molecular thermal motion of urea at 1260 and $123 \mathrm{k}$ from neutron diffraction," Acta Crystallographica, Section B: Structural Science, vol. 40, pp. 300-306, 1984. 3

[26] B. Rousseau, C. V. Alsenoy, R. Keuleers, and H. O. Desseyn, "Solids modeled by ab-initio crystal field methods. part 17. study of the structure and vibrational spectrum of urea in the gas phase and in its crystal phase," J. Phys. Chem., vol. 102, pp. 6540-6548, 1998. 3. 13

[27] R. J. Meier and B. Coussens, "The molecular structure of the urea molecule: Is the minimum energy structure planar?," J. Mol. Struct., vol. 253, pp. 25-33, 1992. 3, 7, 15

[28] A. Gobbi and G. Frenking, "Y-conjugated compounds: the equilibrium geometries and electronic structures of guanidine, guanidinium cation, urea, and 1,1-diaminoethylene," Journal of the American Chemical Society, vol. 115, pp. 2362-2372, 1993. 3, 5,43 
[29] M. Spoliti, A. Pieretti, L. Bencivenni, and N. Sanna, "Theoretical study of the stable c2 and cs symmetry isomers of urea," Electronic Journal of Theoretical Chemistry, vol. 2, pp. 149-159, 1997. 3.5

[30] S. T. King, "Low temperature matrix isolation study of hydrogen-bonded, high-boiling organic compounds iii infrared spectra of monomeric acetamide, urea and urea-d4," Spectrochimica Acta, Part A: Molecular and Biomolecular Spectroscopy, vol. 28, pp. 165-175, 1972. 3

[31] R. D. Brown, P. D. Godfrey, and J. Storey, "The microwave spectrum of urea," J. Mol. Spectrosc., vol. 58, pp. 445-450, 1975. 3.21

[32] P. D. Godfrey, R. D. Brown, and A. N. Hunter, "The shape of urea," J. Mol. Struct., vol. 413-414, pp. 405-414, 1997. 3, 5, 7, 13, 33

[33] J. C. Dobrowolski, R. Kolos, J. Sadlej, and A. P. Mazurek, "Theoretical and ir-matrix isolation studies on the urea and urea-d4, -13c, and -1,3-15n2 substituted molecules," Vibrational Spectroscopy, vol. 29, pp. 261-282, 2002. 3, 13, 33

[34] U. Kaatze, H. Gerke, and R. Pottel, "Dielectric relaxation in aqueous solutions of urea and some of its derivatives," Journal of Physical Chemistry, vol. 90, p. 54645469, 1986. 3

[35] A. Rupprecht and U. Kaatze, "Solution properties of urea and its derivatives in water evidence from ultrasonic relaxation spectra," Journal of Physical Chemistry A, vol. 106, pp. $8850-8858,2002.3$

[36] J. M. Hermida-Ramn, A. hrn, and G. Karlstrm, "Planar or nonplanar what is the structure of urea in aqueous solution," Journal of Physical Chemistry B, vol. 111, pp. 11511-11515, 2007. 3

[37] F. Ramondo, L. Bencivenni, R. Caminiti, A. Pieretti, and L. Gontrani, "Dimerisation of urea in water solution a quantum mechanical investigation," Physical Chemistry Chemical Physics, vol. 9, pp. 2206-2215, 2007. 3 . 5

[38] A. Masunov and J. J. Dannenberg, "Theoretical study of urea. i. monomers and dimers," Journal of Physical Chemistry A, vol. 103, pp. 178-184, 1999. 5 
[39] M. Spoliti, G. Perrone, L. Bencivenni, A. Pieretti, A. Grandi, and F. Ramondo, "Computational and vibrational spectroscopy study of the microclusters of $\mathrm{c} 2$ symmetry urea molecule in the 1a electronic ground state," Journal of Molecular Structure, vol. 756, pp. 113-126, 2005. 5

[40] T. Strassner, "Ab initio and molecular mechanics calculations of various substituted ureas - rotaional barriers and a new parametrization for ureas," Journal of Molecular Modeling, vol. 2, pp. 217-226, 1996. 5, 7, 43

[41] V. S. Bryantsev, T. K. Firman, and B. P. Hay, "Conformational analysis and rotational barriers of alkyl- and phenyl-substituted urea derivatives," Journal of Physical Chemistry A, vol. 109, pp. 832-842, 2005. 5, 7. 43

[42] Y. Zhao, M. K. Raymond, H. Tsai, and J. D. Roberts, "A proton nmr investigation of rotation about the c(o)-n bonds of urea," Journal of Physical Chemistry, vol. 97, pp. 29102913, 1993. 7. 43

[43] J. Langer, B. Schrader, V. Bastian, and E. Jacob, "Infrared spectra and force constants of urea in the gaseous phase," Fresenius' Journal of Analytical Chemistry, vol. 352, pp. 489495, 1995. 9.27

[44] M. W. Schmidt, "General atomic and molecular electronic structure system," Journal of Computational Chemistry, vol. 14, pp. 1347-1363, 1993. 11

[45] J. Thom H. Dunning, "Gaussian basis sets for use in correlated molecular calculations. i. the atoms boron through neon and hydrogen," Journal of Chemical Physics, vol. 90, pp. 1007-1023, 1989. 11

[46] R. A. Kendall, T. H. D. Jr., and R. J. Harrison, "Electron affinities of the first-row atoms revisited. systematic basis sets and wave functions," Journal of Chemical Physics, vol. 96, pp. $6796-6806,1992.11$

[47] C. Möller and M. S. Plesset, "Note on an approximation treatment for many-electron systems," Physical Review, vol. 46, pp. 618-622, 1934. 11, 14

[48] J. N. Murrell and K. J. Laidler, "Symmetries of activated complexes," Transactions of the Faraday Society, vol. 64, pp. 371-377, 1968. 17 
[49] P. Derreumaux, G. Vergoten, and P. Lagant, "A vibrational molecular force field of model compounds with biological interest. i. harmonic dynamics of crystalline urea at $123 \mathrm{k}$," Journal of Computational Chemistry, vol. 11, pp. 560-568, 1990. 20, 43

[50] A. Horwitz, "A version of simpson's rule for multiple integrals," Journal of Computational and Applied Mathematics, vol. 134, pp. 1-11, 2001. 20

[51] W. R. Gilkerson and K. K. Srivastava, "The dipole moment of urea," Journal of Physical Chemistry, vol. 64, p. 14851487, 1960. 21

[52] J. C. Decius, "A tabulation of general formulas for inverse kinetic energy matrix elements in acyclic molecules," Journal of Chemical Physics, vol. 16, pp. 1025-1034, 1948. 27

[53] X. Li, S. J. Stotesbury, and U. A. Jayasooriya, "Infrared spectra of urea isolated in a solid argon matrix," Spectrochimica Acta, Part A: Molecular and Biomolecular Spectroscopy, vol. 43, pp. 1595-1597, 1987. 31

[54] F. Lecomte, B. Lucas, G. Grégoire, J. P. Schermann, and C. Desfrancois, "Urea and methylurea dipole-bound anions," Physical Chemistry Chemical Physics, vol. 5, pp. 31203125, 2003. 33

[55] P. Stilbs and S. Forsen, "Nuclear magnetic resonance study of hindered internal rotation in urea in solution," Journal of Physical Chemistry, vol. 75, pp. 1901-1902, 1971. 43

[56] M. Kontoyianni and J. P. Bowen, "An ab initio and molecular mechanical investigation of ureas and amide derivatives," J. Comput. Chem., vol. 13, pp. 657-666, 1992. 43

[57] D. A. Dixon and N. Matsuzawa, "Density functional study of the structures and nonlinear optical properties of urea," Journal of Physical Chemistry, vol. 98, pp. 3967-3977, 1994. 43

[58] H. C. Longuet-Higgins, "The symmetry groups of non-rigid molecules," Molecular Physics, vol. $6: 5$, pp. $445-460,1963.47$

[59] A. I. B. Veronika V. Nefedova and J. Simons, "Graphical description of the symmetries of potential energy surfaces," Journal of Chemical Physics, vol. 98, pp. 8801-8809, 1993. 55. 59 